\title{
Comparison of SUSY spectrum calculations and impact on the relic density constraints from WMAP
}

\author{
G. Bélanger ${ }^{1}$, S. Kraml ${ }^{2}$, A. Pukhov ${ }^{3}$ \\ 1) Laboratoire de Physique Théorique LAPTH, F-74941 Annecy-le-Vieux, France \\ 2) CERN, Dep. of Physics, Theory Division, CH-1211 Geneva 23, Switzerland \\ 3) Skobeltsyn Inst. of Nuclear Physics, Moscow State Univ., Moscow 119992, Russia
}

\begin{abstract}
We compare results of four public supersymmetric (SUSY) spectrum codes, ISA JET 7 .71, SOFTSUSY 1.9, SPHENO 2.2.2 and SUSPECT 2.3 to estimate the presentday uncertainty in the calculation of the relic density of dark matter in mSUGRA models. We find that even for mass differences of about $1 \%$ the spread in the obtained relic densities can be $10 \%$. In difficult regions of the parameter space, such as large $\tan \beta$ or large $m_{0}$, discrepancies in the relic density are much larger. We also find important differences in the stau co-annihilation region. We show the impact of these uncertainties on the bounds from WMAP for several scenarios, concentrating on the regions of parameter space most relevant for collider phenomenology. We also discuss the case of $A_{0} \neq 0$ and the stop co-annihilation region. Moreover, we present a web application for the online comparison of the spectrum codes.
\end{abstract}

\section{Introduction}

Since the extremely precise measurement of the cosmic microwave background by the WMAP experiment [1, 2], cosmology has been used to severely constrain models with cold dark matter candidates. The prime example are supersymmetric models with Rparity conservation where the neutralino LSP (lightest supersymmetric particle) is the cold dark matter (see Ref. [3] for a review of SUSY cosmology). Requiring that the model provide the right amount of cold dark matter

$$
0.0945 \leq \Omega_{C D M} h^{2} \leq 0.1287
$$

at $2 \sigma$ puts strong constraints on the parameter space of the model, in particular in the mSUGRA scenario $[4,5,6,7,8,9,10,11]$. Effectively, the relic density of dark matter imposes some very specific relations among the parameters of the model. Naturally, the question arises how precisely $\Omega^{1}$ is calculated in a supersymmetric model. We therefore revisit the constraints from WMAP in the mSUGRA scenario taking into account uncertainties originating from the computation of the SUSY spectrum. In the standard approach, the relic density is $\Omega \propto 1 /\langle\sigma v\rangle$, where $\langle\sigma v\rangle$ is the thermally averaged cross section times the relative velocity of the LSP pair. This thermally averaged effective annihilation cross section includes a sum over all annihilation channels for the LSP as well as

\footnotetext{
${ }^{1}$ In what follows, $\Omega \equiv \Omega_{C D M} h^{2}$.
} 
co-annihilation channels involving sparticles that are close in mass to the LSP. The relic density then depends on all the parameters of the MSSM (i.e. masses and couplings) that enter the different annihilation/co-annihilation channels. To calculate the relevant cross sections within the context of a model defined at a high scale, say the GUT scale, one first needs to solve the renormalization group equations to obtain the MSSM parameters at the SUSY scale. Second, higher-order corrections to the masses and couplings need to be calculated. Many public or private spectrum calculators perfom this task. The results are then used to calculate in an improved tree-level approximation the effective annihilation cross-section of neutralinos and the relic density of dark matter. This kind of top-down approach is also the typical method to test high-scale models at the LHC [12]. To address the issue of the precision of the relic density computation in mSUGRA, in this note we compare the results of four public spectrum codes, ISAJET 7.71 [13], SOFTSUSY 1.9 [14], SPHENO 2.2.2 [15] and SUSPECT 2.3 [16], linking them to micrOMEGAs 1.3.2 [17] to compute $\Omega$. Since three of these codes, ISAJET 7.71, SOFTSUSY 1.9 and SPHENO 2.2.2, are of a comparable level as what concerns radiative corrections, the differences in their results seem to be a good estimate of the present uncertainties due to higher-order loop effects. We also include SUSPECT 2.3 in the discussion because it is a widely used program. However, since in contrast to the other three codes, SUSPECT 2.3 has only 1-loop renormalization group ( $\mathrm{RG}$ ) running for the squark and slepton mass parameters we do not use it for the estimate of uncertainties. Within a given program, one can also estimate the theoretical uncertainty by, for example, varying the scale $M_{S U S Y}$ at which the electroweak-symmetry breaking conditions are imposed and the sparticle masses are calculated. This was discussed in Ref. [18] and uncertainties on the relic density up to $20 \%$ were found.

The MSSM parameters that enter the effective annihilation cross section for the LSP include all the ones contributing to the annihilation and co-annihilation processes. The relic density can then depend on a large number of parameters. However, because one needs, at least within the context of SUGRA models, very specific mechanisms to satisfy the tight upper bound of WMAP, only a few parameters are critical within each scenario [18]. Any shift in one of the critical variable can have a large impact on the value of the relic density. Within mSUGRA, the preferred scenarios are the $\tilde{\tau}$ co-annihilation, the rapid Higgs annihilation and the higgsino-LSP scenarios. The main channels are annihilation of neutralinos into fermion pairs via s-channel $\mathrm{Z}$ or Higgs exchange, or via t-channel sfermion exchange, as well as co-annihilation with sleptons. For example, in the co-annihilation region, co-annihilation processes are suppressed by a factor $\exp ^{-\Delta M / T_{f}}$ where $\Delta M$ is the mass difference with the LSP and $T_{f} \approx m_{\tilde{\chi}_{1}^{0}} / 25$ is the decoupling temperature. Then it is the mass difference between the NLSP and LSP that introduces the largest uncertainty in the prediction of the relic density. In Ref. [18] it was shown that a $1 \mathrm{GeV}$ correction to the mass difference could lead to $10 \%$ correction on the relic density. In [19] it has been pointed out that typical differences in the masses obtained by the spectrum calculator codes are of $\mathcal{O}(1 \%)$, large enough for the computational uncertainty to exceed the experimental one of WMAP. In other scenarios, the ones where annihilation proceeds through s-channel $\mathrm{Z}$ or Higgs exchange, the important parameters are the coupling of neutralinos to the $\mathrm{Z}$ or Higgs and the mass of the LSP in relation with the mass of the resonance, in general the mass of the pseudoscalar. These processes are often relevant in the same "tricky" region of parameter space where the discrepancies in the predictions of the spectrum calculators well exceed the $1 \%$ level, Ref. [19], leading to large uncertainties in the relic 
density prediction.

The influence of these differences on relic density computations has first been studied in [20] for the Les Houches 2003 workshop. Since then, all above mentioned programs have undergone major updates; a re-analysis of the existing uncertainties therefore seems appropriate. Moreover, the study of [20] concentrated on potentially large differences along specific lines in the focus point, large $\tan \beta$ and co-annihilation regions. In this article, we consider the WMAP allowed parameter region in the $m_{0}-m_{1 / 2}$ plane, investigating in particular differences in WMAP constraints which arise from the different SUSY spectrum codes. We also address the issue of non-zero $A_{0}$, which for very large $A_{0}<0$ leads to $\tilde{t}$ co-annihilation.

We first briefly discuss in Section 2 the calculation of the supersymmetric spectrum. We then study in Section 3 some specific scenarios: in Section 3.1 we discuss the case of moderate parameters (small $m_{0}$, small to medium $m_{1 / 2}$, moderate $\tan \beta$ ), which is most promising for collider phenomenology and where the calculations are expected to be quite precise. As it turns out there are, however, non-negligible uncertainties already in this region. In Section 3.2, we discuss the case of large $\tan \beta$, where much larger differences are observed. Section 3.3 then deals with the case of large $m_{0}$ and Section 3.4 with the case of large $m_{0}$ and large $\tan \beta$. Here very large uncertainties are found; in particular focus point behaviour may or may not occur depending on the program. The influence of the $A_{0}$ parameter is discussed in Section 3.5. In Section 4, we present a web application for online spectrum comparisons. Finally, Section 5 contains conclusions and an outlook.

For the sake of a fair comparison, we use the same Standard Model (SM) input parameters in all programs. In particular, we use $m_{b}\left(m_{b}\right)^{\overline{M S}}=4.214 \mathrm{GeV}$ and $\alpha_{s}\left(M_{Z}\right)^{\overline{M S}}=$ 0.1172 according to ISAJET 7.71. Moreover, we use a top pole mass of $m_{t}=175 \mathrm{GeV}$ throughout the paper. The parameters of the MSSM are defined following the SUSY Les Houches Accord (SLHA) [21].

We do not discuss here the impact of different cosmological scenarii. We assume the standard cosmological scenario, in particular that at the freeze-out temperature when the interaction rate of particles drops below the expansion rate of the universe, the universe was radiation dominated. Modifications of the standard picture for the expansion of the universe could significantly affect the estimation of the relic density, examples are models with a low-reheating temperature [22] or with scalar-field kination [23].

\section{SUSY spectrum and relic density}

To derive the relic density within a specific SUSY model, mSUGRA for instance, one needs to compute the mass spectrum and couplings from high-energy input parameters. We use the latest version of the four public codes ISAJET 7.71, SOFTSUSY 1.9, SPHENO 2.2.2 and SUSPECT 2.3 for this task and compare their spectra and the resulting neutralino relic densities. These codes basically work as follows: after specifying the gauge and Yukawa couplings in the $\overline{\mathrm{DR}}$ scheme at the electroweak scale and starting with an initial guess of the MSSM parameters, renormalization group ( $R G$ ) equations are used to run the parameters to some high scale $M_{X}$. There boundary conditions are imposed on the SUSY-breaking parameters, and the couplings and parameters are run down to the SUSY mass scale. At that scale radiative electroweak symmetry breaking is checked. The SUSY spectrum is calculated and radiative corrections are computed. The process is repeated 
iteratively until a stable solution is found. The four programs differ, however, in the implementation of radiative corrections (a detailed comparison of the codes can be found in [19]). For one, ISAJET 7.71, SOFTSUSY $1.9^{2}$ and SPHENO 2.2.2 apply full 2-loop RG running for all SUSY mass parameters, while SUSPECT 2.3 calculates gaugino and Higgs mass parameters at 2-loops but squark and slepton parameters only at 1-loop. Second, ISAJET 7.71 uses step beta functions when passing thresholds in the RG evolution, adding additional finite corrections at the end. In contrast to that the other programs compute the complete 1-loop threshold corrections at the SUSY mass scale $M_{S U S Y}=\sqrt{m_{\tilde{t}_{1}} m_{\tilde{t}_{2}}}$. Third, the use of either on-shell or running masses in the loops can significantly influence the results even though the difference is formally a higher-order effect. Moreover, different approximations are used in some parts of the loop corrections. For example, ISAJET 7.71 and SPHENO 2.2.2 apply the complete 1-loop corrections given in [24] for the neutralino and stau masses, while SOFTSUSY 1.9 and SUSPECT 2.3 use the approximate expressions of [24] for neutralinos and do not include the self-energies for the staus. The calculation of the light Higgs mass has recently been standardized between SOFTSUSY 1.9, SPHENO 2.2.2 and SUSPECT 2.3 to full 1-loop plus leading 2-loop corrections, see [25]. ISAJET 7.71 on the other hand uses an 1-loop effective potential, which typically leads to about $2-3 \mathrm{GeV}$ higher $h^{0}$ masses compared to the other programs. Notice, however, that this lies within the present $\sim 2-3 \mathrm{GeV}$ theoretical uncertainty in $m_{h}$. Moreover, as we will see, the exact value of $m_{h}$ is only important in a narrow strip in the large $m_{0}$ region. All considered, we take ISAJET 7.71, SOFTSUSY 1.9 and SPHENO 2.2.2 as being of a comparable level of sophistication as concerns the SUSY and heavy Higgs masses. Two-loop as opposed to one-loop scalar running, as in SUSPECT 2.3, can however have an important influence on the relic density through differences in the sfermion masses.

The nature of the LSP, which is a linear combination of the bino $\tilde{B}$, wino $\tilde{W}$ and the two higgsino states $\tilde{H}_{1,2}$, is a crucial parameter in the evaluation of the relic density;

$$
\tilde{\chi}_{1}^{0}=N_{11} \tilde{B}+N_{12} \tilde{W}+N_{13} \tilde{H}_{1}+N_{14} \tilde{H}_{2}
$$

where $N$ is the neutralino mixing matrix. The LSP-higgsino fraction is given as

$$
f_{H}=N_{13}^{2}+N_{14}^{2}
$$

and is large when the higgsino mass parameter $\mu \lesssim M_{1}, M_{2}$, where $M_{1}$ and $M_{2}$ are the $\mathrm{U}(1)$ and $\mathrm{SU}(2)$ gaugino masses. The LSP coupling to the pseudoscalar, $g_{\tilde{\chi}_{1}^{0} \tilde{\chi}_{1}^{0} A}$, depends on the same elements of the neutralino mixing matrix:

$$
g_{\tilde{\chi}_{1}^{0} \tilde{\chi}_{1}^{0} A} \propto N_{13}^{2}-N_{14}^{2} .
$$

The value of $\mu$ depends sensitively, in certain regions of parameter space, on the SM input parameters, in particular the top quark mass and its relation with the top Yukawa couplings. At large $m_{0}$, the top Yukawa coupling has a strong influence on $m_{H_{2}}^{2}$; as a result the $\mu$ parameter becomes very sensitive to $h_{t}=\sqrt{2} \hat{m}_{t} / v_{2}$, where $\hat{m}_{t}$ is the running t-quark mass and $v_{2}$ is the vev of the second Higgs doublet:

$$
\mu^{2}=\frac{\left(\bar{m}_{H_{1}}^{2}-\bar{m}_{H_{2}}^{2} \tan ^{2} \beta\right)}{\tan ^{2} \beta-1}-\frac{1}{2} M_{Z}^{2}
$$

\footnotetext{
${ }^{2}$ Here note that the default option in SOFTSUSY 1.9 is 1-loop running of the squark and slepton mass parameters; 2-loop running of these parameters has to be switched on by hand. In the following, we always take SOFTSUSY 1.9 with full 2-loop RGE running.
} 
Here $\bar{m}_{H_{i}}^{2}=m_{H_{i}}^{2}-t_{i} / v_{i}, i=1,2$, with $t_{i}$ the tadpole contributions. See [19] for more detail. For the intermediate to large values of $\tan \beta$ that we will consider, the $m_{H_{2}}^{2}$ term dominates in the extraction of $\mu$. Differences in the $\mu$ parameter will affect the neutralino couplings, in particular the coupling to the pseudoscalar $A$. In the mass spectrum these differences most obviously show up as differences in the mass of the neutralino that is dominantly higgsino, usually $\tilde{\chi}_{3}^{0}$. The programs under consideration all apply the 1-loop corrections of [24] plus the 2-loop QCD corrections of [26]. Nevertheless the differences in $h_{t}$ are large enough to lead to huge discrepancies in $\mu$ at large $m_{0}$.

The mass of the pseudoscalar, $m_{A}$, is another important parameter in the computation of the relic density. This mass also depends sensitively on the SM input parameters, in particular the bottom quark mass and its translation to the bottom Yukawa coupling. The bottom Yukawa coupling which is large at high $\tan \beta$ impacts the Higgs sector since $m_{H_{1}}^{2}$ is driven by $h_{b}=\sqrt{2} \hat{m}_{b} / v_{1}$, where $\hat{m}_{b}$ is the running b-quark mass and $v_{1}$ is the vev of the first Higgs doublet. The physical pseudoscalar mass directly depends on $m_{H_{1}}^{2}$ :

$$
m_{A}^{2}=\frac{1}{\cos 2 \beta}\left(\bar{m}_{H_{1}}^{2}-\bar{m}_{H_{2}}^{2}\right)+\frac{s_{\beta}^{2} t_{1}}{v_{1}}+\frac{c_{\beta}^{2} t_{2}}{v_{2}}-M_{Z}^{2}
$$

The four spectrum codes all apply the corrections of $[24,26]$, resumming the 1-loop SUSY corrections according to [27]. This brings in general good agreement on $m_{A}$; however as we will see the remaining differences can still lead to sizable discrepancies in $\Omega$ in parts of the parameter space.

\section{Results}

\subsection{Small $m_{0}$, small to medium $m_{1 / 2}$, moderate $\tan \beta$}

We start out with an easy, collider-friendly scenario of small $m_{0}$, small to medium $m_{1 / 2}$ and moderate $\tan \beta$. Such a scenario has gluinos and squarks with masses up to $1 \mathrm{TeV}$ which cascade-decay into neutralinos and sleptons. It can hence provide the favourite LHC signature of jets plus same-flavor opposite-sign leptons. It also has gauginos and sleptons within the kinematical reach of a future $e^{+} e^{-}$linear collider (ILC) and is thus very well suited for both LHC and ILC studies.

In the region considered in this section, as in most of the mSUGRA parameter space, the LSP is nearly a pure bino. As such it couples preferably to right-chiral sfermions with a coupling proportional to the hypercharge. The main annihilation channel for the LSP is then into lepton pairs via t-channel exchange of right-chiral sleptons. This process is efficient enough to meet the WMAP upper limit only in the low $m_{0}-m_{1 / 2}$ corner of the parameter space, the so-called bulk region. Indeed, for a pure bino LSP the relic density is approximately $\Omega \propto m_{\tilde{l}_{R}}^{4} / m_{\tilde{\chi}_{1}^{0}}^{2}$, implying that both the $\tilde{l}_{R}$ and the $\tilde{\chi}_{1}^{0}$ must be light. Since sleptons must be beyond the reach of LEP2, the upper limit from WMAP is only satisfied in a very small region below $m_{1 / 2} \sim 240 \mathrm{GeV}$, see Fig. 1 . The bulk region is, however, associated with a light Higgs below the LEP2 limit ${ }^{3}$. Light neutralinos can also annihilate efficiently into fermion pairs near a $Z$ or Higgs resonance. This corresponds to the near vertical WMAP line in Fig. 1. This possibility is however by large excluded by

\footnotetext{
${ }^{3}$ Note that an increase in the top-quark mass loosens the LEP2 constraint from the light Higgs.
} 


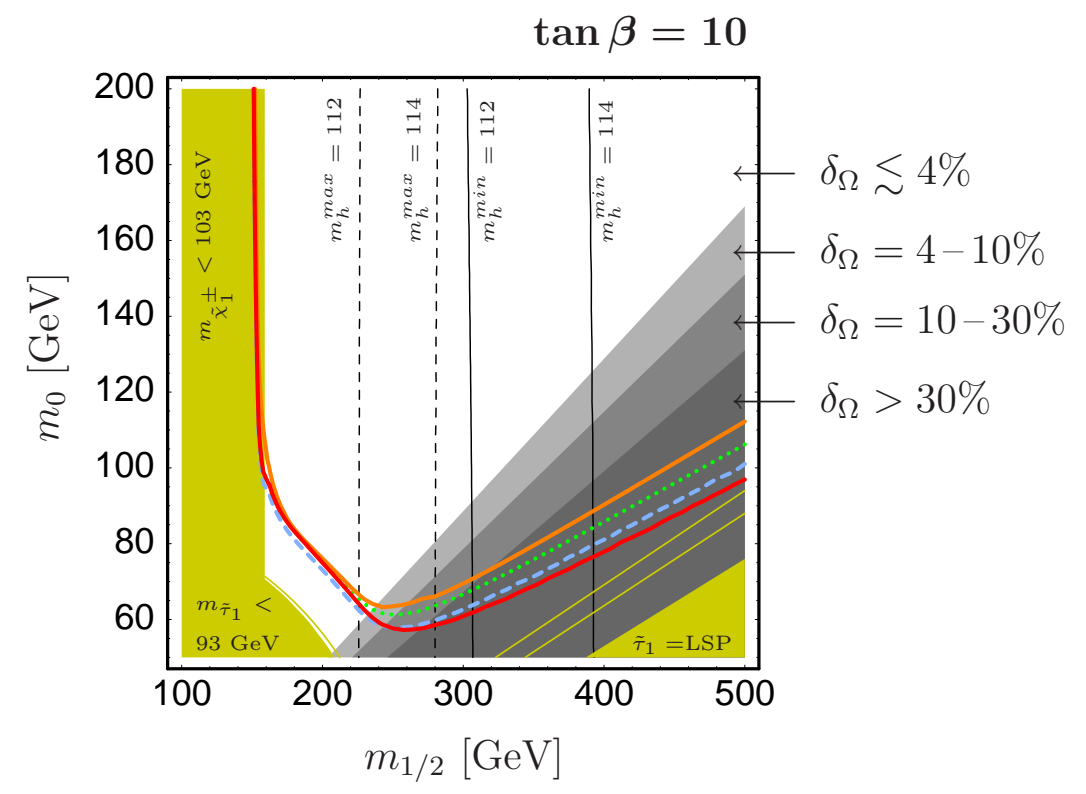

Figure 1: Comparison of results in the $m_{0}-m_{1 / 2}$ plane, for $A_{0}=0, \tan \beta=10, \mu>0$, and $m_{t}=175 \mathrm{GeV}$. The red (dark) and orange (light) full lines show the variation of the $2 \sigma$ upper limit $\Omega<0.1287$ when micrOMEGAs 1.3 .2 is linked to ISAJET 7.71 , SOFTSUSY 1.9 or SPHENO 2.2.2. The orange line basically comes from SOFTSUSY 1.9 while the red one comes from ISAJET 7.71. In addition, the upper bound from SPHENO 2.2.2 is shown as green dotted line, and that of SUSPECT 2.3 as blue dashed line. The light, medium and dark gray shaded areas show the regions where the relative differences in $\Omega, \delta \Omega$ of eq. (7), are $4-10 \%, 10-30 \%$ and $>30 \%$, respectively. Also shown are contours of minimal (full black lines) and maximal (dashed black lines) $h^{0}$ masses as obtained by the spectrum codes. The yellow region on the left is excluded by LEP2 constraints; in the yellow triangle in the bottom right corner $m_{\tilde{\tau}_{1}}<m_{\tilde{\chi}_{1}^{0}}$ in ISAJET 7.71. The yellow lines show the boundaries of the excluded region in the other codes.

the LEP direct limits [28] on chargino pairs, which in effect translate into a lower limit on the LSP mass in mSUGRA.

Agreement with WMAP is recovered for heavier neutralinos $\left(m_{1 / 2} \gtrsim 240 \mathrm{GeV}\right)$ with the additional contributions from co-annihilation channels, the so-called co-annihilation region. For co-annihilation to be effective, the mass difference between the slepton NLSP and the $\tilde{\chi}_{1}^{0}$ LSP must be rather small (less than $\sim 10 \mathrm{GeV}$ ). Such degenerate sleptons/neutralinos are found in the low $m_{0}$ region of mSUGRA. The $\tilde{\tau}_{1}$ is the lightest slepton due both to the effect of the $\tau$-Yukawa coupling in the RGE running of $m_{\tilde{\tau}_{R}}$ as well as to the mixing between $\tilde{\tau}_{L}$ and $\tilde{\tau}_{R}$ which lowers the mass of the $\tilde{\tau}_{1}$ to $m_{\tilde{\tau}_{1}}<m_{\tilde{\tau}_{R}}$. In fact, co-annihilation processes with $\tilde{\tau}_{1}$ dominate over most of the allowed region in Fig. 1. In the co-annihilation region, it was shown in Ref. [18] that the relic density is extremely sensitive to the mass difference between $\tilde{\tau}_{1}$ and $\tilde{\chi}_{1}^{0}, \Delta M\left(\tilde{\chi}_{1}^{0} \tilde{\tau}_{1}\right)$. Typically a shift in $\Delta M\left(\tilde{\chi}_{1}^{0} \tilde{\tau}_{1}\right) \approx 1 \mathrm{GeV}$ induces $\Delta \Omega \approx 10 \%$. Previous comparisons between the public SUSY spectrum codes [19] have shown that the predicted masses often differ by more than $\pm 1 \%$, inducing discrepancies in $\Delta M\left(\tilde{\chi}_{1}^{0} \tilde{\tau}_{1}\right)$ above $\pm 1 \mathrm{GeV}$ and hence large uncertainties in the relic density.

These expectations are corroborated by a scan in the $m_{0}-m_{1 / 2}$ plane comparing the predictions of the four spectrum codes. Figure 1 shows results for $A_{0}=0, \tan \beta=10$, 
$\mu>0$ and $m_{t}=175 \mathrm{GeV}$. The red and orange lines show the variation of the $2 \sigma$ upper limit $\Omega<0.1287$ when micrOMEGAs 1.3 .2 is linked to ISAJET 7.71 , SOFTSUSY 1.9 and SPHENO 2.2.2. In addition, the light, medium and dark gray shaded areas show the regions where the relative differences in $\Omega$,

$$
\delta_{\Omega} \equiv\left(\Omega_{\text {max }}-\Omega_{\text {min }}\right) / \Omega_{\text {mean }},
$$

are $4-10 \%, 10-30 \%$ and $>30 \%$, respectively. Here $\Omega_{\max }$ and $\Omega_{\min }$ are the maximal and minimal values and $\Omega_{\text {mean }}$ the arithmetic mean of the $\Omega$ values obtained from ISAJET 7.71, SOFTSUSY 1.9 and SPHENO 2.2.2 at a specific parameter point. We do not include SUSPECT 2.3 in the calculation of $\delta_{\Omega}$ because it has only 1-loop scalar running. A $\delta_{\Omega}$ of $30 \%$ corresponds to the present precision of WMAP, while the PLANCK experiment [29] is expected to reach a precision of $4 \%$, corresponding to the white area in Fig. 1. Also shown are contours of the minimal and maximal $h^{0}$ masses as obtained by the four spectrum codes. As a general rule, the $m_{h}^{\max }$ lines come from ISAJET 7.71, while the $m_{h}^{\min }$ lines come from the other programs. Note that the bulk region is practically excluded.

The red (maximal $\Omega$ ) and orange (minimal $\Omega$ ) lines in Fig. 1 come from ISAJET 7.71 and SOFTSUSY 1.9, respectively. The values obtained from SPHENO 2.2.2, shown as dotted green line, lie in between these curves. In the co-annihilation region the results of SUSPECT 2.3, shown as dashed blue line, fall within the red and orange lines. While the differences in the WMAP bounds in Fig. 1 do not look dramatic, it becomes clear from the grey shaded areas that the relative differences in $\Omega$ are quite large in the allowed parameter space, that is in the co-annihilation region, where the precise mass differences, in particular between $\tilde{\tau}_{1}$ and $\tilde{\chi}_{1}^{0}$, are important.

ISAJET 7.71, SOFTSUSY 1.9 and SPHENO 2.2.2 typically agree on the $\tilde{\tau}_{1}$ mass to $\sim 1 \%$. The difference mainly comes from SOFTSUSY 1.9, which neglects the tau Yukawa coupling $h_{\tau}$ and the $\tilde{\tau}$ self-energy correction and hence gets a slightly smaller $m_{\tilde{\tau}_{1}}$. SOFTSUSY 1.9 , SPHENO 2.2.2 and SUSPECT 2.3 agree very well on the $\tilde{\chi}_{1}^{0}$ mass, while ISAJET 7.71 finds a $m_{\tilde{\chi}_{1}^{0}}$ smaller by about $2 \%$. As a consequence, both SOFTSUSY 1.9 and SPHENO 2.2.2 tend to give a smaller $\tilde{\tau}_{1}-\tilde{\chi}_{1}^{0}$ mass difference than the other two programs, and hence a smaller $\Omega$ in the co-annihilation region. As an example, Table 1 lists the relevant masses together with $\Omega$ for $m_{0}=70 \mathrm{GeV}, m_{1 / 2}=350 \mathrm{GeV}, A_{0}=0, \tan \beta=10$ and $\mu>0$. Table 2 gives the according relative contributions to $\Omega^{-1}$ for this point. Note here the contribution of the $\tilde{\tau}_{1}$ and $\tilde{e_{R}}, \tilde{\mu}_{R}$ co-annihilation channels. Clearly our expectations that the mass difference is the most important parameter are confirmed. The $2 \mathrm{GeV}$ decrease in $\Delta M\left(\tilde{\chi}_{1}^{0} \tilde{\tau}_{1}\right)$ when going from SPHENO 2.2.2 to SOFTSUSY 1.9 roughly corresponds to a decrease of $\mathcal{O}(20 \%)$ in $\Omega$ as expected. As a result of the mass spectrum, one finds a larger contribution from the co-annihilation channels for SOFTSUSY 1.9 where it amounts to almost $80 \%$ of the effective annihilation cross section as compared to the other codes where co-annihilation channels contribute $\sim 50-70 \%$. ISAJET 7.71 , which agrees well with SPHENO 2.2.2 on the $\tilde{\tau}_{1}$ mass but finds a smaller $m_{\tilde{\chi}_{1}^{0}}$, has the largest $\tilde{\chi}_{1}^{0}-\tilde{\tau}_{1}$ mass difference and a $\sim 50 \%$ higher $\Omega$ as compared to SPHENO 2.2.2. For similar $\Delta M\left(\tilde{\chi}_{1}^{0} \tilde{\tau}_{1}\right)$, ISAJET 7.71 predicts a slightly lower value for the relic density as compare to other codes because of a lower LSP mass. SUSPECT 2.3 on the other hand agrees well with SOFTSUSY 1.9/SPHENO 2.2.2 on the LSP mass, but due to the missing 2-loop effects in the running of the slepton masses it gets a heavier $\tilde{\tau}_{1}$ (and $\tilde{e}_{R}$ ) and hence a larger $\Omega$. We have checked that when using only 1loop RGEs for the slepton mass parameters in SOFTSUSY 1.9, it reproduces the results of SUSPECT 2. 3. 


\begin{tabular}{c|cccc} 
& ISA JET 7 .71 & SOFTSUSY 1.9 & SPHENO 2.2.2 & SUSPECT 2.3 \\
\hline$\tilde{\chi}_{1}^{0}$ & 136.7 & 140.0 & 139.5 & 140.0 \\
$\tilde{\tau}_{1}$ & 147.7 & 145.7 & 147.1 & 149.7 \\
$\tilde{e}_{R}$ & 155.7 & 153.8 & 155.4 & 157.6 \\
$h^{0}$ & 115.8 & 113.1 & 113.4 & 113.3 \\
\hline$m_{\tilde{\tau}_{1}}-m_{\tilde{\chi}_{1}^{0}}$ & 11.0 & 5.7 & 7.6 & 9.7 \\
\hline$\Omega$ & 0.136 & 0.069 & 0.092 & 0.120 \\
\hline
\end{tabular}

Table 1: Relevant masses, the $\tilde{\chi}_{1}^{0}-\tilde{\tau}_{1}$ mass difference (in $\mathrm{GeV}$ ) and the resulting $\Omega$ for $m_{0}=70 \mathrm{GeV}, m_{1 / 2}=350 \mathrm{GeV}, A_{0}=0, \tan \beta=10$ and $\mu>0$. The higgsino fraction of $\tilde{\chi}_{1}^{0}$ is $1.4-1.5 \%$ in all cases.

\begin{tabular}{l|cccc} 
channel & ISAJET 7.71 & SOFTSUSY 1.9 & SPHENO 2.2.2 & SUSPECT 2.3 \\
\hline$\tilde{\chi}_{1}^{0} \tilde{\chi}_{1}^{0} \rightarrow e e$ & $28 \%$ & $10 \%$ & $16 \%$ & $22 \%$ \\
$\tilde{\chi}_{1}^{0} \tilde{\chi}_{1}^{0} \rightarrow \tau \tau$ & $16 \%$ & $6 \%$ & $9 \%$ & $13 \%$ \\
$\tilde{\chi}_{1}^{0} \tilde{e}_{R} \rightarrow \gamma / Z e$ & $8 \%$ & $8 \%$ & $8 \%$ & $10 \%$ \\
$\tilde{\chi}_{1} \tilde{\tau}_{1} \rightarrow \gamma / Z \tau$ & $30 \%$ & $39 \%$ & $38 \%$ & $34 \%$ \\
$\tilde{\tau}_{1} \tilde{\tau}_{1} \rightarrow \tau \tau$ & $5 \%$ & $17 \%$ & $11 \%$ & $7 \%$ \\
$\tilde{\tau}_{1} \tilde{\tau}_{1} \rightarrow \gamma \gamma, \gamma Z$ & $2 \%$ & $7 \%$ & $6 \%$ & $3 \%$ \\
$\tilde{e}_{R} \tilde{\tau}_{1} \rightarrow e \tau$ & $2 \%$ & $6 \%$ & $4 \%$ & $2 \%$ \\
\hline
\end{tabular}

Table 2: Relative contributions to $\Omega^{-1}$ for $m_{0}=70 \mathrm{GeV}, m_{1 / 2}=350 \mathrm{GeV}, A_{0}=0$, $\tan \beta=10$ and $\mu>0$; with $e \equiv e, \mu$ and $\tilde{e}_{R} \equiv \tilde{e}_{R}, \tilde{\mu}_{R}$.

Some more comments are in order. First, a non-zero value of $A_{0}$ shifts the contours of constant Higgs masses and moves the position of the stau co-annihilation strips as well as of the excluded regions in Fig. 1; it does however not change the picture qualitatively, provided $A_{0}$ is not so large as to make $\tilde{t}_{1}$ the (N)NLSP. The case of a non-zero $A_{0}$ will be discussed in detail in Section 3.5. Second, for the reference point SPS1a' of the SPA project [30], $\left(m_{0}=70 \mathrm{GeV}, m_{1 / 2}=250 \mathrm{GeV}, A_{0}=-300, \tan \beta=10, \mu>0\right.$ and $m_{t}=178 \mathrm{GeV}$ ), ISAJET 7.71, SOFTSUSY 1.9 and SPHENO 2.2.2 give values of $\Omega=0.126$, 0.103 and 0.114 , respectively. SOFTSUSY 1.9 with 1 -loop scalar running gives $\Omega=0.125$, and SUSPECT $2.3 \Omega=0.126$. All values lie within the WMAP allowed range of Eq. (1) at this point, with the spread of $\delta \Omega \simeq 20 \%$ again being mainly due to $\Delta M\left(\tilde{\chi}_{1}^{0} \tilde{\tau}_{1}\right)$.

\section{$3.2 \quad$ Large $\tan \beta$}

We next consider large values for $\tan \beta$; we stay however within collider-friendly scenarios with small $m_{0}$ and small to medium $m_{1 / 2}$. At large values of $\tan \beta$, the enhanced couplings of the heavy Higgses to $b \bar{b}$ and $\tau \tau$ lead to an enhancement of neutralino annihilation channels through $\tilde{\chi}_{1}^{0} \tilde{\chi}_{1}^{0} \rightarrow H^{0}, A^{0} \rightarrow b \bar{b}, \tau \tau$. Because of the Majorana nature of the LSP the main contribution is the pseudoscalar exchange, the CP-even state being Pwave suppressed. Even though the LSP is mostly bino, its small higgsino component is sufficient to make annihilation through the pseudoscalar and the Goldstone component of $Z$ exchange dominant. These contributions are added to the contributions from t-channel 
sfermion exchange or from co-annihilation with staus that were already present at lower values of $\tan \beta$.

At low $m_{1 / 2}$, the annihilation into $b \bar{b}$ typically constitutes more than $80 \%$ of the effective annihilation cross section. For a fixed value of $m_{1 / 2}$, hence of neutralino mass, the relic density decreases with $m_{0}$ since both the sfermion masses as well as the pseudoscalar mass decrease, making for more efficient annihilation. Because of the enhanced contribution of the pseudoscalar exchange, a much larger region of parameter space in the bulk is compatible with the WMAP upper bound as compared to intermediate $\tan \beta$ values, see Fig. 2. Nevertheless as one moves towards larger values of $m_{1 / 2}$ and a heavier LSP, one must again appeal to co-annihilation to retain consistency with WMAP, leading to a mixed region with both co-annihilation and pseudoscalar exchange. The co-annihilation occurs exclusively with $\tilde{\tau}_{1}$, which is much lighter than the other sleptons at large $\tan \beta$. Note that for the range of $m_{1 / 2}$ which we are considering, we are never near the heavy Higgs pole.

The relic density is again sensitive to $\Delta M\left(\tilde{\chi}_{1}^{0} \tilde{\tau}_{1}\right)$ for the co-annihilation processes. Sensitivity to $\Delta M\left(\tilde{\chi}_{1}^{0} A\right)=m_{A}-2 m_{\tilde{\chi}_{1}^{0}}$ as well as to the $\tilde{\chi}_{1}^{0} \tilde{\chi}_{1}^{0} A$ coupling are expected for the Higgs contribution, see [18]. As already mentioned, the bottom and tau Yukawa couplings play an important role in radiative corrections to the sparticle and Higgs masses at large $\tan \beta$, leading to larger differences in the spectra. Consequently in the computation of the relic density we also observe larger discrepancies between the four codes.

Figure 2 compares the results of the various codes in the $m_{0}-m_{1 / 2}$ plane analogous to Fig. 1 but for $\tan \beta=40$ (left) and $\tan \beta=50$ (right). The other parameters are $A_{0}=0$, $\mu>0$ and $m_{t}=175 \mathrm{GeV}$ as before. At $\tan \beta=40$, the WMAP exclusion curves seem to agree quite well. Small differences $(\Delta \Omega<5 \%)$ are observed over much of the plane, but these increase rapidly to $10-30 \%$ and more as one moves into the WMAP allowed region. Near the stau-LSP border, differences in the predictions of the spectrum calculators for the $\tilde{\tau}_{1}$ masses, and hence for $m_{\tilde{\tau}_{1}}-m_{\tilde{\chi}_{1}^{0}}$, explain this discrepancy, just as was the case for $\tan \beta=10$. Large differences are also observed for low $m_{1 / 2}$ in the region near the band excluded by LEP limits. These discrepancies are due to differences in $m_{\tilde{\chi}_{1}^{0}}$. Specifically some codes allow a significant annihilation rate through the light Higgs exchange in a region that is allowed by the LEP limit on charginos. Here again the low $m_{1 / 2}$ (bulk) region is not compatible with the lower limit on the Higgs mass.

For further illustration, we pick a parameter point from Fig. $2 \mathrm{a},\left(m_{0}, m_{1 / 2}\right)=(194,300)$ $\mathrm{GeV}$ at $\tan \beta=40$. Details on the spectrum relevant for the relic density calculation and the list of important channels for all four codes are presented in Table 3. For this parameter choice we are in a mixed region where both co-annihilation and Higgs exchange processses are important. All codes agree quite well on the values of $m_{\tilde{\chi}_{1}^{0}}, m_{A}$ and consequently $m_{A}-2 m_{\tilde{\chi}_{1}^{0}}$ with maximal variation on the latter of about $5 \%$. The variation in the $\mu$ parameter is below $3 \%$. The variation of the NLSP-LSP mass difference is $2 \%$ within ISAJET 7.71, SOFTSUSY 1.9 and SPHENO 2.2.2, but $40 \%$ if we also include SUSPECT 2.3. The difference in the $\tilde{\tau}_{1}$ mass between SOFTSUSY 1.9 and SPHENO 2.2.2 can again be explained by the missing $h_{\tau}$ and $\tilde{\tau}$ self-energy corrections in the former program, which is roughly a $1 \%$ effect. It is interesting to note that this also influences $m_{A}$ at the level of few per-mille. All considered, the uncertainties in the Higgs annihilation and the stau co-annhilation channels are of similar importance in ISAJET 7.71, SOFTSUSY 1.9 and SPHENO 2.2.2, leading to a spread in $\Omega$ of $25 \%$ for the parameter point of Table 3 . If we interpret this as $\Omega=0.107 \pm 0.013$, then SUSPECT 2.3 deviates by $2.7 \sigma$ due to its larger 

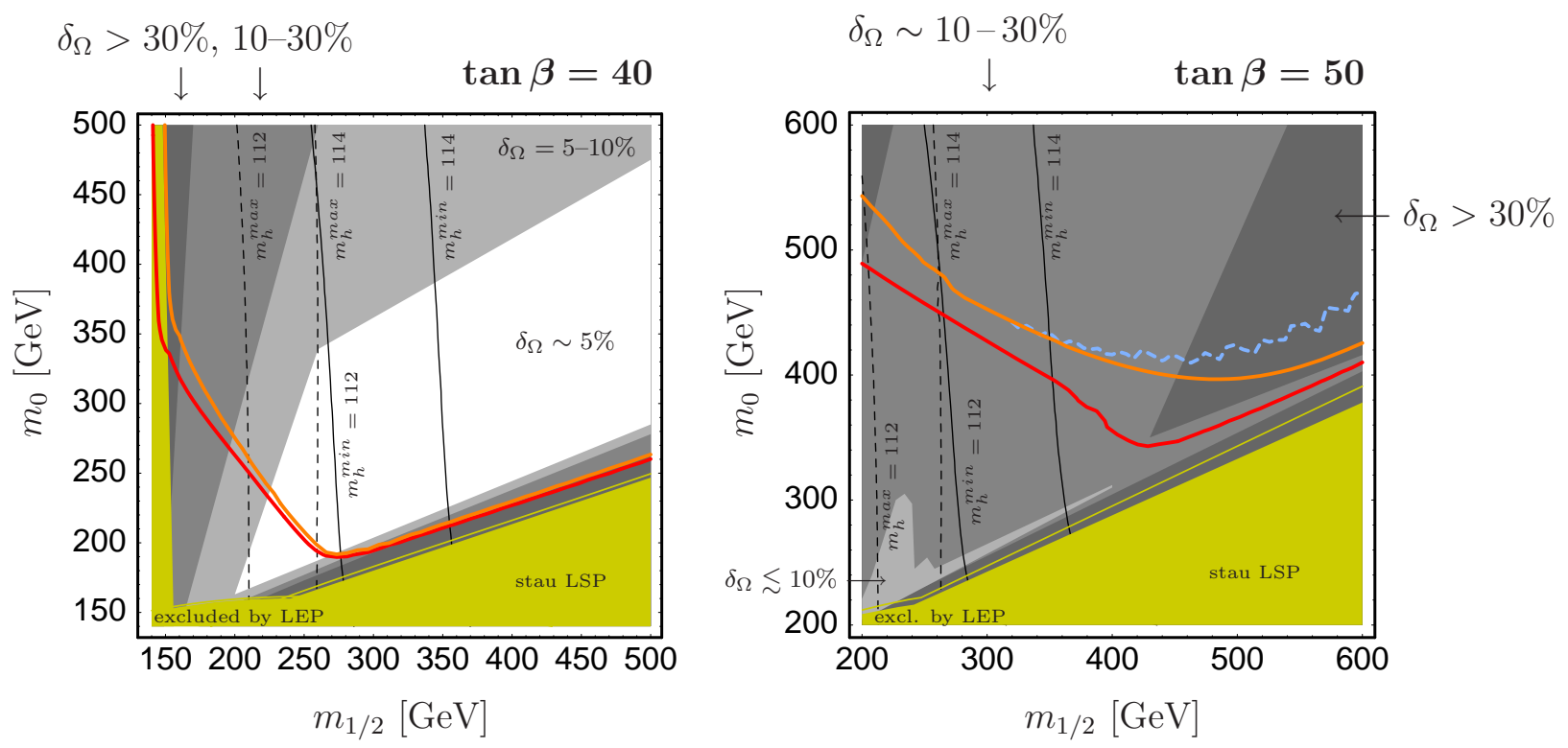

Figure 2: Comparison of results analogous to Fig. 1 but for $\tan \beta=40$ (left) and $\tan \beta=$ 50 (right); $A_{0}=0, \mu>0$, and $m_{t}=175 \mathrm{GeV}$. The red and orange lines show again the variation of the bound $\Omega<0.1287$ due to differences in the spectra from ISAJET 7.71, SOFTSUSY 1.9 and SPHENO 2.2.2. In the right panel, the blue dashed line shows in addition how the upper curve would move when including SUSPECT 2.3.
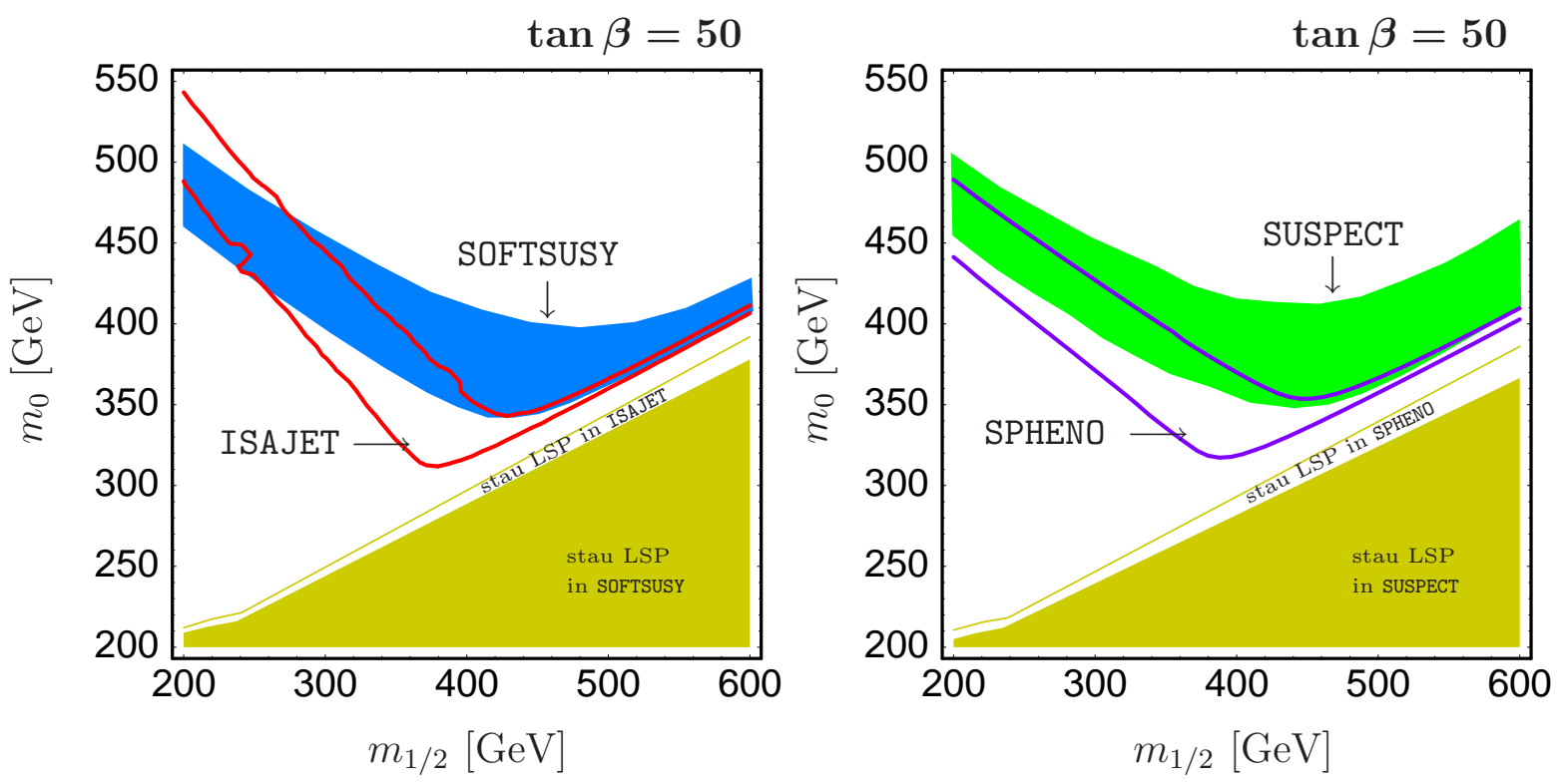

Figure 3: WMAP allowed regions of $0.0945 \leq \Omega \leq 0.1287$ for $\tan \beta=50, A_{0}=0$, $\mu>0, m_{t}=175 \mathrm{GeV}$; left: ISAJET 7.71 and SOFTSUSY 1.9, right: SPHENO 2.2.2 and SUSPECT 2.3. 
NLSP-LSP mass difference.

Increasing further $\tan \beta$ means that the $A b \bar{b}$ coupling is further enhanced and pseudoscalar exchange dominates over most of the probed parameter space. Figure $2 \mathrm{~b}$ compares the various codes for $\tan \beta=50$. In this case, large discrepancies are found in the relic density and this over most of the parameter space. In particular, the minimal and maximal upper boundaries from WMAP displayed as red and orange curves differ significantly. As a result of the pseudoscalar exchange contribution, the bulk region is much larger compared to small $\tan \beta$. We however stress that this bulk region is not one of t-channel sfermion exchange but rather one of heavy Higgs annihilation. Stau coannihilation also plays a role near the stau-NLSP boundary; however this typically drives the relic density below the WMAP range. Large $\tan \beta$ also means larger discrepancies between the predictions of the spectrum calculators especially for the pseudoscalar mass. The large discrepancies in $\Omega$ over most of the parameter space are due to the differences in $m_{A}-2 m_{\tilde{\chi}_{1}^{0}}$. Typically, at such large $\tan \beta$ the mass of the pseudoscalar in SOFTSUSY 1.9 and SUSPECT 2.3 is lighter than that predicted by the other two codes. The only region where $\delta \Omega<10 \%$ lies at small $m_{1 / 2}$ where the pseudoscalar exchange diagram is less important and better agreement for the masses is found.

Figure 3 explicitly compares the $2 \sigma$ WMAP allowed regions of the four programs at $\tan \beta=50$. The difference in the prediction of the pseudoscalar mass also explains why the band near the stau co-annihilation region is much narrower for ISA JET 7.71 and SPHENO 2.2.2. Here one is sitting too far from the Higgs resonance to get a significant contribution to the annihilation cross-section, the only remaining WMAP allowed region being the narrow stau co-annihilation strip. For further illustration, we pick a parameter point from Fig. $3,\left(m_{0}, m_{1 / 2}\right)=(350,350) \mathrm{GeV}$ at $\tan \beta=50$. The results for this point are listed in Table 4. Here the mass difference between the NLSP and the LSP is much too large to get a significant contribution from co-annihilation processes. The main channels are annihilation of neutralinos into fermion pairs via pseudoscalar exchange. Although one is far from the Higgs resonance, this process is efficient enough due to the enhanced coupling of the Higggs to $b \bar{b}$ and $\tau \tau$. For this point one gets as usual rather good agreement among all codes in the $\tilde{\chi}_{1}^{0}$ masses and in the higgsino fraction. The pseudoscalar masses also agree within 1-2\%; for the resonance parameter $m_{A}-2 m_{\tilde{\chi}_{1}^{0}}$ the differences are however around $10 \%$. In [18] it was shown that in this region a $4 \%$ shift in $m_{A}-2 m_{\tilde{\chi}_{1}^{0}}$ leads to a $10 \%$ change in $\Omega$. The discrepancies in the mass difference found in Table 4 explain the difference between the value of the relic density in SPHENO 2.2.2, SOFTSUSY 1.9 and SUSPECT 2.3. In the case of ISAJET 7.71 the decrease in the annihilation cross section due to the fact that one is sitting further away from the Higgs resonance is partly compensated by a lower value of the $\mu$ parameter (to wit the smaller value of $m_{\tilde{\chi}_{3}^{0}}$ ) hence a larger $\tilde{\chi}_{1}^{0} \tilde{\chi}_{1}^{0} A$ coupling. We have also checked explicitly that by adjusting $m_{A}-2 m_{\tilde{\chi}_{1}^{0}}$ to the SPHENO 2.2.2 value in SOFTSUSY 1.9 we recover very good agreement between the two programs.

To put these results in perspective, we also remark that there is a strong $m_{b}\left(m_{b}\right)$ dependence in the computation of the pseudoscalar Higgs mass as discussed in Section 2. This has an impact on the relic density [31, 18]. For example for the parameters of Table 4 , decreasing $m_{b}\left(m_{b}\right)$ to $4.168 \mathrm{GeV}$ (less than a $2 \%$ change) makes the result of SOFTSUSY 1.9 agree perfectly with the ones from ISAJET 7.71. Considering that there are large theoretical uncertainties in the extraction of $m_{b}\left(m_{b}\right)$, this source of uncertainty at present exceeds the one estimated by taking the difference between codes. 
ISAJET 7.71 SOFTSUSY 1.9 SPHENO 2.2.2 SUSPECT 2.3

\begin{tabular}{c||cccc}
\hline \hline$\tilde{\chi}_{1}^{0}$ & 117.2 & 119.9 & 119.7 & 119.9 \\
$\tilde{\tau}_{1}$ & 131.4 & 133.2 & 131.4 & 137.7 \\
$h^{0}$ & 115.3 & 112.7 & 113.0 & 112.8 \\
$A^{0}$ & 363.4 & 363.2 & 366.4 & 364.4 \\
$\tilde{\chi}_{3}^{0}$ & 394.9 & 401.4 & 405.3 & 405.3 \\
\hline$m_{\tilde{\tau}_{1}}-m_{\tilde{\chi}_{1}^{0}}$ & 14.2 & 13.3 & 11.6 & 17.8 \\
$m_{A}-2 m_{\tilde{\chi}_{1}^{0}}$ & 129 & 123 & 127 & 125 \\
\hline$\tilde{\chi}_{1}^{0} \tilde{\chi}_{1}^{0} \rightarrow b b$ & $40 \%$ & $38 \%$ & $30 \%$ & $49 \%$ \\
$\tilde{\chi}_{1}^{0} \tilde{\chi}_{1}^{0} \rightarrow e e$ & $12 \%$ & $10 \%$ & $10 \%$ & $14 \%$ \\
$\tilde{\chi}_{1}^{0} \tilde{\chi}_{1}^{0} \rightarrow \tau \tau$ & $17 \%$ & $14 \%$ & $13 \%$ & $19 \%$ \\
$\tilde{\chi}_{1}^{0} \tilde{\tau}_{1} \rightarrow h \tau$ & $13 \%$ & $16 \%$ & $21 \%$ & $7 \%$ \\
$\tilde{\chi}_{1}^{0} \tilde{\tau}_{1} \rightarrow \gamma / Z \tau$ & $12 \%$ & $14 \%$ & $18 \%$ & $7 \%$ \\
$\tilde{\tau}_{1} \tilde{\tau}_{1} \rightarrow h h$ & $1 \%$ & $2 \%$ & $3 \%$ & - \\
\hline$\Omega$ & 0.120 & 0.107 & 0.094 & 0.142 \\
\hline
\end{tabular}

Table 3: Masses and mass differences (in $\mathrm{GeV}$ ), the most important contributions, and the resulting $\Omega$ for $m_{0}=194 \mathrm{GeV}, m_{1 / 2}=300 \mathrm{GeV}, A_{0}=0, \mu>0$ and $\tan \beta=40$. The higgsino fraction of $\tilde{\chi}_{1}^{0}$ is $1.8 \%$ in all cases.

ISAJET 7.71 SOFTSUSY 1.9 SPHENO 2.2.2 SUSPECT 2.3

\begin{tabular}{c||cccc}
\hline \hline$\tilde{\chi}_{1}^{0}$ & 139.1 & 142.2 & 141.9 & 142.1 \\
$\tilde{\tau}_{1}$ & 208.7 & 217.6 & 214.6 & 223.3 \\
$h^{0}$ & 116.3 & 113.9 & 114.3 & 114.1 \\
$A^{0}$ & 369.0 & 366.2 & 371.9 & 365.3 \\
$\tilde{\chi}_{3}^{0}$ & 449.9 & 457.3 & 462.7 & 463.1 \\
\hline$m_{\tilde{\tau}_{1}}-m_{\tilde{\chi}_{1}^{0}}$ & 70 & 75 & 73 & 81 \\
$m_{A}-2 m_{\tilde{\chi}_{1}^{0}}$ & 91 & 82 & 88 & 81 \\
\hline$\tilde{\chi}_{1}^{0} \tilde{\chi}_{1}^{0} \rightarrow b b$ & $81 \%$ & $83 \%$ & $82 \%$ & $83 \%$ \\
$\tilde{\chi}_{1}^{0} \tilde{\chi}_{1}^{0} \rightarrow \tau \tau$ & $15 \%$ & $14 \%$ & $14 \%$ & $14 \%$ \\
\hline$\Omega$ & 0.104 & 0.087 & 0.102 & 0.088 \\
\hline
\end{tabular}

Table 4: Same as Table 3 but for $m_{0}=m_{1 / 2}=350 \mathrm{GeV}$ and $\tan \beta=50$. The higgsino fraction of $\tilde{\chi}_{1}^{0}$ is $1.4 \%$. 


\subsection{Large $m_{0}$, focus point}

Large $m_{0}$ is a notoriously difficult region which suffers from large uncertainties. The reason is the extreme sensitivity of the $\mu$ parameter to the top Yukawa coupling alluded to in Section 2. We limit our discussion to gaugino and higgsino masses within the reach of LHC and ILC and consider values of $m_{0}$ up to $4.5 \mathrm{TeV}$. Figure 4 shows the allowed regions in the $m_{0}-m_{1 / 2}$ plane for $m_{0}=1-4.5 \mathrm{TeV}, \tan \beta=10, A_{0}=0, \mu>0$ and $m_{t}=175 \mathrm{GeV}$. A striking discrepancy between the codes is the occurrence or non-occurrence of focus-point behaviour and related with this the limit of radiative electroweak symmetry breaking (REWSB). In Fig. 4, the four programs agree more or less up to $m_{0} \sim 2 \mathrm{TeV}$. Above this value, the results of ISAJET 7.71 become very different, with REWSB breaking down around $m_{0} \sim 2.7-3 \mathrm{TeV}$. In SOFTSUSY 1.9 and in SUSPECT 2.3 this happens only around $m_{0} \sim 3.5-4 \mathrm{TeV}$ while in SPHENO 2.2.2 one can go to much higher $m_{0}$. In fact this behaviour is related to small differences in the treatment of the top Yukawa coupling; focus point behaviour can be recovered for all codes when one lowers the top-quark mass.

In the allowed parameter space, the main annihilation channel for neutralinos is into fermion pairs. Consistency with WMAP then requires some enhancement factor for the annihilation cross section. This is in principle provided by the light Higgs resonance but only in a narrow strip of the parameter space. The relic density hence becomes very sensitive to the $m_{h}-2 m_{\tilde{\chi}_{1}^{0}}$ mass difference (as compared to the decoupling temperature of the neutralinos, $\left.T_{f} \approx m_{\tilde{\chi}_{1}^{0}} / 25\right)$. The width of the $h^{0}$ is not an important parameter because it is much smaller then $T_{f}$.

When the $\tilde{\chi}_{1}^{0}$ mass is slightly below half the $h^{0}$ mass, most of the $\tilde{\chi}_{1}^{0}$ 's annihilate efficiently through $\tilde{\chi}_{1}^{0} \tilde{\chi}_{1}^{0} \rightarrow h^{0} \rightarrow b \bar{b}$. This requires a very small $m_{1 / 2}$, roughly $m_{1 / 2} \lesssim 150 \mathrm{GeV}$ as can be seen in Fig. 4. On the other hand, the LEP bound of $m_{\tilde{\chi}_{1}^{ \pm}}>103 \mathrm{GeV}$ requires $m_{1 / 2} \gtrsim 130-140 \mathrm{GeV}$. In Fig. 4 the bands that are within the $2 \sigma$ WMAP range correspond to $m_{h}-2 m_{\tilde{\chi}_{1}^{0}}$ either of a few hundred $\mathrm{MeV}$ or around $10 \mathrm{GeV}$ (15 GeV in case of ISA JET 7.71). In between these values, the Higgs annihilation mechanism is too efficient, resulting in $\Omega<0.0945$. Table 5 gives examples for $m_{0}=2 \mathrm{TeV}$ and $m_{0}=3.8 \mathrm{TeV}$. For $m_{0}=2 \mathrm{TeV}$, SOFTSUSY 1.9, SPHENO 2.2.2 and SUSPECT 2.3 predict similar masses and LSP higgsino fractions. As expected, the relic density decreases as one moves slightly away from the pole. For ISAJET 7.71, predictions for the relic density for a given $m_{h}-2 m_{\tilde{\chi}_{1}^{0}}$ are typically lower than for the other codes, since two other effects enhance the annihilation cross-section: a larger LSP higgsino fraction and the fact that with a lighter LSP one benefits from the Z-exchange contribution. For $m_{0}=3.8 \mathrm{TeV}$, only SOFTSUSY 1.9, SPHENO 2.2.2 and SUSPECT 2.3 find viable RGE solutions. There are now large $\sim 60 \%$ discrepancies in the $\mu$ parameter also among these codes. This is reflected in quite different higgsino fractions, and in turn in $\mathcal{O}(100 \%)$ differences in the values of $\Omega$. As a side remark we note that the large uncertainties in the $\mu$ parameter also lead to significant discrepancies in the $\tilde{\chi}_{3,4}^{0}$ and $\tilde{\chi}_{1,2}^{ \pm}$masses, which can considerably impact the collider phenomenology of a particular mSUGRA point.

Another comment is in order. Within any of the spectrum codes a change in $m_{t}$ of the order of what will be measured at LHC $\left(\Delta m_{t} \sim 1 \mathrm{GeV}\right)$ induces large changes in the value of $\mu$ and hence in the LSP mass, its higgsino fraction, and the relic density. The latter can vary by over an order of magnitude within a given code. This is due to the extreme sensitivity of the running of $m_{\mathrm{H}_{2}}^{2}$ to the top Yukawa coupling as explained in Section 2, c.f. eq. (5). Small changes in the input value of $m_{t}$ can therefore bring approximate 

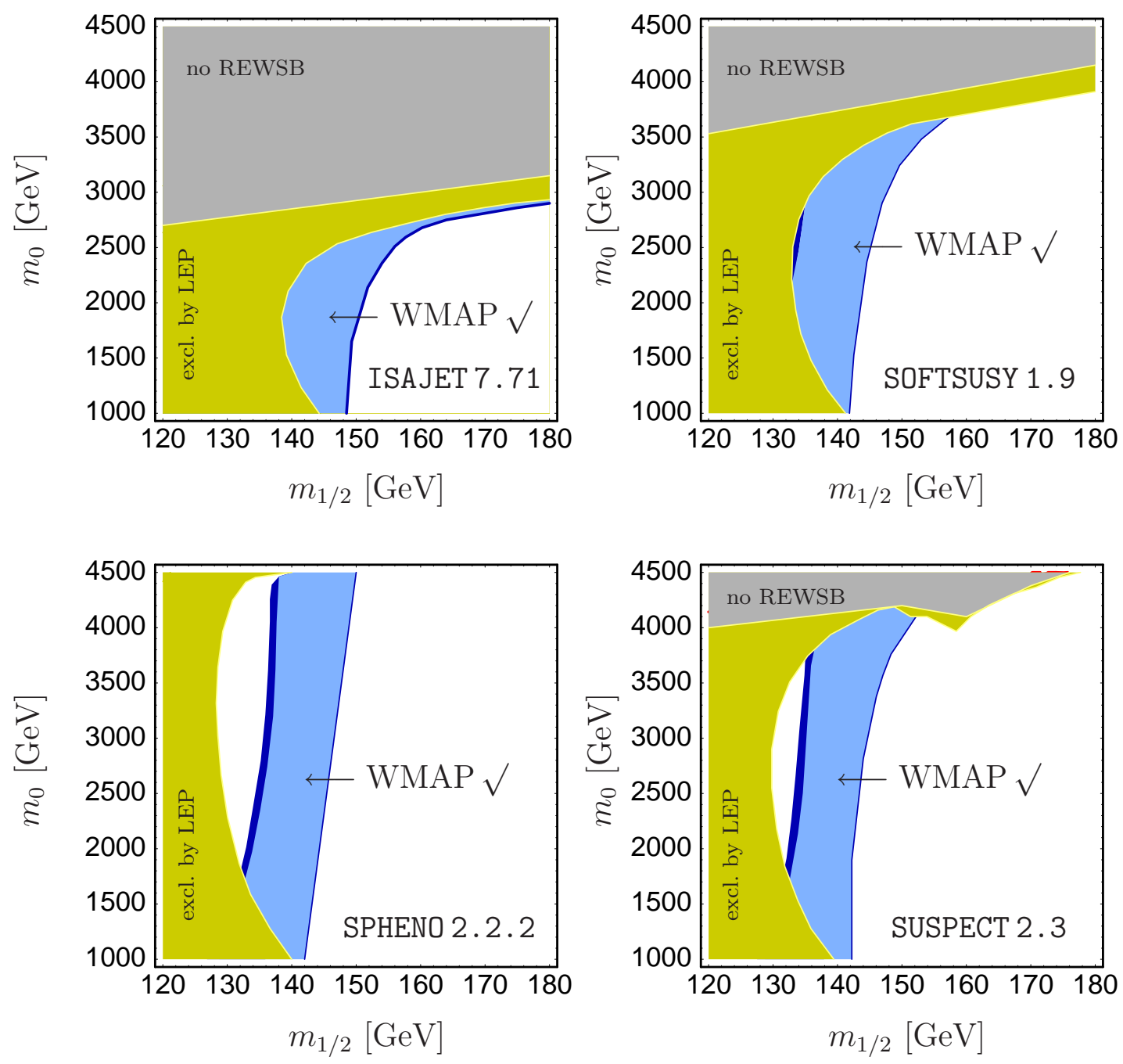

Figure 4: WMAP allowed regions (blue) in the $m_{0}-m_{1 / 2}$ plane for large $m_{0} ; \tan \beta=10$, $A_{0}=0, \mu>0, m_{t}=175 \mathrm{GeV}$. In the dark blue bands $0.0945 \leq \Omega \leq 0.1287$, while in the light blue bands $\Omega<0.0945$. In the gray areas there is no radiative EWSB; the yellow regions are excluded by the LEP bound $m_{\tilde{\chi}_{1}^{ \pm}}>103 \mathrm{GeV}$. 


\begin{tabular}{|c|c|c|c|c|}
\hline \multicolumn{5}{|c|}{$m_{0}=2 \mathrm{TeV}$} \\
\hline & ISAJET 7.71 & SOFTSUSY 1.9 & SPHENO 2. 2.2 & SUSPECT 2.3 \\
\hline$\tilde{\chi}_{1}^{0}$ & 54.9 & 57.8 & 58.2 & $\begin{array}{l}58.2 \\
\end{array}$ \\
\hline$h^{0}$ & 115.9 & 116.5 & 116.9 & 116.7 \\
\hline$\tilde{\chi}_{3}^{0}$ & 290.5 & 383.9 & 450.6 & 441.5 \\
\hline$m_{h}-2 m_{\tilde{\chi}_{1}^{0}}$ & 6.1 & 0.9 & 0.5 & 0.3 \\
\hline$f_{H}\left(\tilde{\chi}_{1}^{0}\right)$ & $4.0 \%$ & $1.9 \%$ & $1.3 \%$ & $1.3 \%$ \\
\hline$\tilde{\chi}_{1}^{0} \tilde{\chi}_{1}^{0} \rightarrow b b$ & $90 \%$ & $90 \%$ & $90 \%$ & $90 \%$ \\
\hline$\tilde{\chi}_{1}^{0} \tilde{\chi}_{1}^{0} \rightarrow \tau \tau$ & $9 \%$ & $9 \%$ & $9 \%$ & $9 \%$ \\
\hline$\Omega$ & 0.011 & 0.011 & 0.023 & 0.038 \\
\hline \multicolumn{5}{|c|}{$m_{0}=3.8 \mathrm{TeV}$} \\
\hline & ISAJET 7.71 & SOFTSUSY 1.9 & SPHENO 2. 2.2 & SUSPECT 2.3 \\
\hline$\tilde{\chi}_{1}^{0}$ & - & 56.1 & (59.2 & 57.8 \\
\hline$h^{0}$ & - & 125.8 & 122.1 & 121.6 \\
\hline$\tilde{\chi}_{3}^{0}$ & - & 243.7 & 450.7 & 301.9 \\
\hline$m_{h}-2 m_{\tilde{\chi}_{1}^{0}}$ & - & 13.6 & 3.7 & 6.0 \\
\hline$f_{H}\left(\tilde{\chi}_{1}^{0}\right)$ & - & $6.2 \%$ & $1.3 \%$ & $3.4 \%$ \\
\hline$\tilde{\chi}_{1}^{0} \tilde{\chi}_{1}^{0} \rightarrow b b$ & - & $82 \%$ & $90 \%$ & $90 \%$ \\
\hline$\tilde{\chi}_{1}^{0} \tilde{\chi}_{1}^{0} \rightarrow \tau \tau$ & - & $9 \%$ & $9 \%$ & $9 \%$ \\
\hline$\Omega$ & - & 0.066 & 0.021 & 0.012 \\
\hline
\end{tabular}

Table 5: Relevant masses and mass differences (in $\mathrm{GeV}$ ), the higgsino fraction of the LSP, the most important contributions and the resulting $\Omega$ for $m_{0}=2$ and $3.8 \mathrm{TeV}$, $m_{1 / 2}=144 \mathrm{GeV}$, and $\tan \beta=10\left(A_{0}=0, \mu>0, m_{t}=175 \mathrm{GeV}\right)$.

agreement between the different codes. We emphazise however that this only reflects the large theoretical uncertainty in this regime.

\subsection{Large $m_{0}$, large $\tan \beta$}

As we increase $\tan \beta$ it becomes increasingly easier to reach the focus point region. There is also a strong dependence on the value of the top-quark mass, and typically ISAJET 7.71 can find a focus point behaviour with significantly heavier $m_{t}$ than the other codes [20]. We consider in more details the case $\tan \beta=50$ and $m_{t}=175 \mathrm{GeV}$. A value for the relic density in agreement with WMAP requires $M_{1}<\mu<M_{2}$ so that the LSP is a mixed bino-higgsino state. As one moves very close to the electroweak symmetry breaking border and $\mu$ drops even below $M_{1}$, the higgsino fraction increases rapidly; the relic density drops below the WMAP range. In what follows we concentrate again on collider-friendly scenarios with not so heavy neutralinos and charginos.

At large $\tan \beta$ and large $m_{0}$, the main neutralino annihilation channels are into fermion pairs or into pairs of gauge or Higgs bosons. Fermion pair production proceeds through s-channel exchange of Higgs or Z (the Goldstone component) and is proportionnal to the fermion mass. Annihilation into $t t$ is therefore favoured as soon as it becomes kinematically accessible. If not, $\mathrm{W}$-pair production is the dominant channel, proceeding via 


\begin{tabular}{c||ccc} 
& SOFTSUSY 1.9 & SPHENO 2.2.2 & SUSPECT 2.3 \\
\hline \hline$\tilde{\chi}_{1}^{0}$ & 135.0 & 148.9 & 146.5 \\
$\tilde{\chi}_{1}^{ \pm}$ & 184.0 & 287.0 & 256.0 \\
$\tilde{\chi}_{2}^{0}$ & 195.9 & 286.9 & 257.4 \\
$\tilde{\chi}_{3}^{0}$ & 212.9 & 502.7 & 324.5 \\
$h^{0}$ & 121.6 & 122.2 & 121.6 \\
$A^{0}$ & 1200 & 1425 & 957 \\
\hline$f_{H}\left(\tilde{\chi}_{1}^{0}\right)$ & $30 \%$ & $1.1 \%$ & $4.3 \%$ \\
\hline$\tilde{\chi}_{1}^{0} \tilde{\chi}_{1}^{0} \rightarrow b b$ & $5 \%$ & $27 \%$ & $44 \%$ \\
$\tilde{\chi}_{1}^{0} \tilde{\chi}_{1}^{0} \rightarrow \tau \tau$ & - & $4 \%$ & $6 \%$ \\
$\tilde{\chi}_{1}^{0} \tilde{\chi}_{1}^{0} \rightarrow Z Z$ & $18 \%$ & $7 \%$ & $6 \%$ \\
$\tilde{\chi}_{1}^{0} \tilde{\chi}_{1}^{0} \rightarrow W W$ & $61 \%$ & $29 \%$ & $21 \%$ \\
$\tilde{\chi}_{1}^{0} \tilde{\chi}_{1}^{0} \rightarrow Z h$ & $8 \%$ & $15 \%$ & $10 \%$ \\
$\tilde{\chi}_{1}^{0} \tilde{\chi}_{1}^{0} \rightarrow h h$ & $5 \%$ & $15 \%$ & $10 \%$ \\
\hline$\Omega$ & 0.125 & 18.6 & 2.15 \\
\hline
\end{tabular}

Table 6: Relevant masses (in GeV), the higgsino fraction of the LSP, the most important contributions and the resulting $\Omega$ for $m_{0}=3450 \mathrm{GeV}, m_{1 / 2}=350 \mathrm{GeV}, \tan \beta=50$, $A_{0}=0, \mu>0$.

t-channel exchange of charginos. Neutralino/chargino co-annihilation channels can be important as well, but typically they are so efficient that they lead to $\Omega$ below the WMAP range. The $\mu$ parameter, which determines the neutralino and chargino masses as well as the $\tilde{\chi}_{1}^{0} \tilde{\chi}_{1}^{0} Z / A$ coupling, also has a significant influence on the relic density. In [18] it was shown that $\Delta \mu \approx 1-2 \%$ could induce shifts of $10 \%$ in $\Omega$ for $\tan \beta=50$. The dependence on $m_{\tilde{\chi}_{1}^{0}}$ or on the pseudoscalar mass is expected to be weaker; corrections of $50 \%$ or larger are necessary to induce a $10 \%$ shift in $\Omega$ [18].

We consider more closely the point $m_{0}=3450 \mathrm{GeV}, m_{1 / 2}=350 \mathrm{GeV}$ and $\tan \beta=50$. Table 6 displays the results for the spectrum and the most important contributions. For this scenario SOFTSUSY 1.9 gives a result within the WMAP range. ISAJET 7.71, however, does not find a solution to the RGEs; the relic density of SPHENO 2.2.2 and SUSPECT 2.3 is orders of magnitude above the WMAP bound. The reason for the latter is that the $\mu$ parameter in SUSPECT 2.3 and even more in SPHENO 2.2.2 is much larger and one is in a regime of a mostly bino LSP -hence no efficient channel for annihilation is available (c.f. the values for $m_{\tilde{\chi}_{3}^{0}}$ and $f_{H}\left(\tilde{\chi}_{1}^{0}\right)$ in Table 6 ). Moreover, SOFTSUSY 1.9 predicts much lighter charginos, which makes annihilation into $\mathrm{W}$ pairs through chargino exchange more efficient. Owing to the huge $\tilde{\chi}_{1}^{0}-A^{0}$ mass difference, the influence of the pseudoscalar mass on the relic density is small, although there is a spread in the prediction of $m_{A}$ of several hundred GeV. This discrepancy in $m_{A}$ becomes, however, very relevant in the Higgs funnel region, that is for larger values of $m_{1 / 2}$.

As was the case in the previous section, within any of the spectrum codes a small change in $m_{t}$ induces large changes in the value of $\mu$ and hence the relic density, which can vary as before by over an order of magnitude within a given code. Using a different input value for $m_{t}$ can therefore compensate the large discrepancies observed between different codes. For example, a decrease of about $0.5 \mathrm{GeV}$ in $m_{t}$ brings the results of SUSPECT 2.3 for both the spectrum and the relic density, in good agreement with those of 
SOFTSUSY 1.9. SOFTSUSY 1.9's results of Table 6 can also be approximately reproduced with ISAJET 7.71 using $m_{t}=176.36 \mathrm{GeV}$. Note however that this amounts to extreme fine-tuning.

\subsection{Varying $A_{0}$}

Non-zero values of $A_{0}$ can significantly influence the scalar masses as well as the $\mu$ parameter. Roughly speaking, for $A_{0}<0{ }^{4}$ the $\tilde{t}_{1}, \tilde{b}_{1}, \tilde{\tau}_{1}$ masses decrease while $m_{A}$ and $\mu$ increase. For $A_{0}>0$, the shifts go in the opposite directions. The pseudoscalar mass is relevant for annihilation processes at large $\tan \beta$ where $\tilde{\chi}_{1}^{0} \tilde{\chi}_{1}^{0} \rightarrow A^{0} \rightarrow f \bar{f}$. The $\mu$ parameter determines the higgsino fraction of the LSP. It also directly influences the mixing in the stau sector and therefore the contribution of the $\tilde{\tau}$ coannihilation processes. With our convention, $A_{0}=0$ leads to $A_{t}<0$ at the weak scale; $A_{0}<0$ increases $\left|A_{t}\right|$ (at the weak scale) thus lowering the $\tilde{t}_{1}$ mass through i) RG running and ii) a larger $\tilde{t}_{L}-\tilde{t}_{R}$ mixing. Analogous arguments hold for sbottoms and staus, though here the $\mathrm{L}-\mathrm{R}$ mixing is dominated by $\mu \tan \beta$. Also the running of $A_{\tau}$ is less strong, so that $A_{\tau}$ usually does not change sign with respect to $A_{0}$. The masses and trilinear couplings of the third generation enter in turn the running of the Higgs mass parameters, the radiative corrections to the Higgs pole masses, and the computation of $\mu$.

The uncertainties in the masses, estimated as the differences between the codes, tend to be larger for $A_{0} \neq 0$ as compared to $A_{0}=0$. Nevertheless the general picture outlined in the previous sections holds, as the same mechanisms as for $A_{0}=0$ are at work for neutralino (co-)annihilation over most of the parameter space. Only when $\left|A_{0}\right|$ becomes large enough to make $\tilde{t}_{1}$ very light, in fact the NLSP or NNLSP, new co-annihilation channels appear associated with a new region of parameter space where the relic density is consistent with WMAP.

Let us discuss the cases of moderate and large $\tan \beta$ in more detail. For $\tan \beta=10$, a non-zero value of $A_{0}$ shifts the contours of constant light Higgs masses (towards lower values of $m_{1 / 2}$ for $A_{0}<0$ ) and moves the position of the stau co-annihilation strips as well as of the excluded regions (towards higher values of $m_{0}$ for $A_{0}<0$ ) as compared to Fig. 1. It does however not change the picture qualitatively; the WMAP allowed regions are a small bulk region with $\tilde{\chi}_{1}^{0} \tilde{\chi}_{1}^{0}$ annihilation and a narrow strip of co-annihilation with staus. There is an increase in the differences in $m_{\tilde{\tau}_{1}}-m_{\tilde{\chi}_{1}^{0}}$ and hence in $\Omega$ between the codes, but the effect is in general not very large. The only new feature appears for values of $\left|A_{0}\right|$ large enough to make $\tilde{t}_{1}$ the $(\mathrm{N})$ NLSP. This case will be illustrated later in this section.

For $\tan \beta=50$, we observe larger discrepancies between the codes even for moderate values of $A_{0}$. This is not surprising as the mixing in the $\tilde{\tau}$ sector depends on $\mu \tan \beta$ and relatively small shifts in $\mu$ can have important effects on the $\tilde{\tau}_{1}$ mass. Moreover, the pseudoscalar mass $m_{A}$ is quite sensitive to $A_{0}$. While for $A_{0}=0$ (and small to medium $m_{0}$ ) ISAJET 7.71, SOFTSUSY 1.9, SPHENO 2.2.2 and SUSPECT 2.3 typically agree on $m_{A}$ to $1-2 \%$, for $A_{0} \neq 0$ differences of a few per-cent can show up. Figure 5 compares the regions of the $m_{0}-m_{1 / 2}$ plane compatible with the upper limit of WMAP analogous to Fig. $2 \mathrm{~b}$ but for $A_{0}=m_{1 / 2}$. As can be seen, the WMAP bound is shifted towards higher values of $m_{0}$. This is because, as mentionned above, the pseudoscalar mass decreases

\footnotetext{
${ }^{4}$ Using SLHA conventions, the off-diagonal element of the $\{$ up, down $\}$-type sfermion mass matrix is $m_{L R}^{2}=\left(A_{f}-\mu\{\cot \beta, \tan \beta\}\right) m_{f}$.
} 
with increasing $A_{0}$, so annihilation channels through Higgs exchange are favoured. The Higgs exchange dominates over most of the region of the plot, however with rather large differences in $\Omega$. The largest differences are found for $m_{1 / 2} \gtrsim 400 \mathrm{GeV}$, as was the case for $A_{0}=0$. Differences in the pseudoscalar masses increase with increasing $A_{0}$ and $m_{1 / 2}$, with SOFTSUSY 1.9 and SUSPECT 2.3 predicting smaller $m_{A}$ than SPHENO 2.2 .2 and ISAJET 7.71 for $A_{0}=m_{1 / 2} \gtrsim 300 \mathrm{GeV}$ (for smaller values, it is ISAJET 7.71, which predicts the lightest $m_{A}$ ). ISAJET 7.71 also predicts a lighter LSP and a lighter $\tilde{\tau}_{1}$ in the co-annihilation range and hence a much lower $\Omega$ as one moves closer to the $\tilde{\tau}_{1}$-LSP boundary. SUSPECT 2.3 on the other hand predicts larger $\tilde{\tau}_{1}$ but smaller pseudoscalar masses than the other programs. This leads to a larger value for $\Omega$ from the SUSPECT 2.3 spectrum for $A_{0}=m_{1 / 2} \gtrsim 400 \mathrm{GeV}$. In Fig. 5, the dashed blue line shows how the exclusion curve correponding to the maximal $\Omega$ is shifted when including SUSPECT 2.3. One can conclude that for $\tan \beta=50$ differences between the codes are large everywhere, with $\delta \Omega$ exceeding $30 \%$ in a large portion of parameter space.

We have also studied the case $A_{0}=-m_{1 / 2}$ at $\tan \beta=50$. Here the pseudoscalar mass is larger than in the $A_{0}=0$ case, so a relic density in agreement with WMAP requires, especially at large $m_{1 / 2}$, some contribution from co-annihilation processes, in particular with $\tilde{\tau}_{1}$. Therefore the value of the relic density is once again very sensitive to the $\tilde{\chi}_{1}^{0}-\tilde{\tau}_{1}$ mass difference, and discrepancies in $\Omega$ are larger than for $A_{0}=0$. Since for $A_{0}<0$ we encounter instabilities in the scan with SPHENO 2.2.2, we do not show a plot but examplify this case in Table 7 for $m_{0}=376 \mathrm{GeV}, m_{1 / 2}=-A_{0}=400 \mathrm{GeV}$, $\tan \beta=50$ and $\mu>0$. Here the discrepancy in the $\tilde{\tau}_{1}$ mass reaches about $10 \%$, meaning that the $\tilde{\chi}_{1}^{0}-\tilde{\tau}_{1}$ mass difference varies by more than $100 \%$, thus inducing huge differences in the relic density. The lightest $\tilde{\tau}_{1}$ is again obtained with ISAJET 7.71. We do however find rather good agreement between the codes as concerns the boundary of the WMAP region.

A special case is a very large negative $A_{0}$, such that $\tilde{t}_{1}$ becomes light enough to contribute to co-annihilations. This is the case when the $\tilde{t}_{1}$ is the NNLSP or even the NLSP. The relic density is then very sensitive to the mass difference between $\tilde{\chi}_{1}^{0}$ and $\tilde{t}_{1}$. Since the largest discrepancies between spectrum calculators are usually found for the masses of coloured sparticles [19], the predictions for the relic density and for the region compatible with WMAP can differ significantly in this case. Figure 6 shows the WMAP-allowed strips in the $m_{0}-m_{1 / 2}$ plane for $A_{0}=-4 m_{1 / 2}, \tan \beta=10$ and $\mu>0$. For ISAJET 7.71, SOFTSUSY 1.9 and SPHENO 2.2.2, co-annihilation with stops dominates when $m_{1 / 2} \lesssim 350-400 \mathrm{GeV}$, while for larger $m_{1 / 2}$ one has mostly stau co-annihilation. For SUSPECT 2.3, $\tilde{t}_{1}$ co-annihilation dominates over the whole allowed region. As expected, the allowed bands are very narrow. They correspond to $m_{\tilde{t}_{1}}-m_{\tilde{\chi}_{1}^{0}} \approx 20-30 \mathrm{GeV}$, typically a much larger mass difference than for the case of $\tilde{\tau}$ co-annihilation. This is due to the large cross section of $\tilde{\chi}_{i}^{0} \tilde{t}_{1} \rightarrow t h, t g$. Table 8 shows the spectrum as well as the most important contributions to $\Omega$ for one point, $m_{0}=161 \mathrm{GeV}, m_{1 / 2}=350 \mathrm{GeV}, A_{0}=-1400 \mathrm{GeV}$, $\tan \beta=10$ and $\mu>0$. As one can see, SOFTSUSY 1.9 and SPHENO 2.2.2 agree quite well on the $\tilde{t}_{1}$ mass and hence the relic density, with only few per-cent difference between the two programs. In comparison, ISAJET 7.71 predicts a lighter $\tilde{t}_{1}$ and thus a much smaller relic density at a given parameter point. The difference is at the level of $10 \%$ for $m_{\tilde{t}_{1}}$ and of $\mathcal{O}(100 \%)$ for $\Omega$. Also the boundaries where $\tilde{t}_{1}$ becomes the LSP are quite different between SOFTSUSY 1.9/SPHENO 2.2.2 on the one side and ISAJET 7.71 on the other side. Part of the discrepancies may come from large logs in the RGEs in ISAJET 7.71 due to 


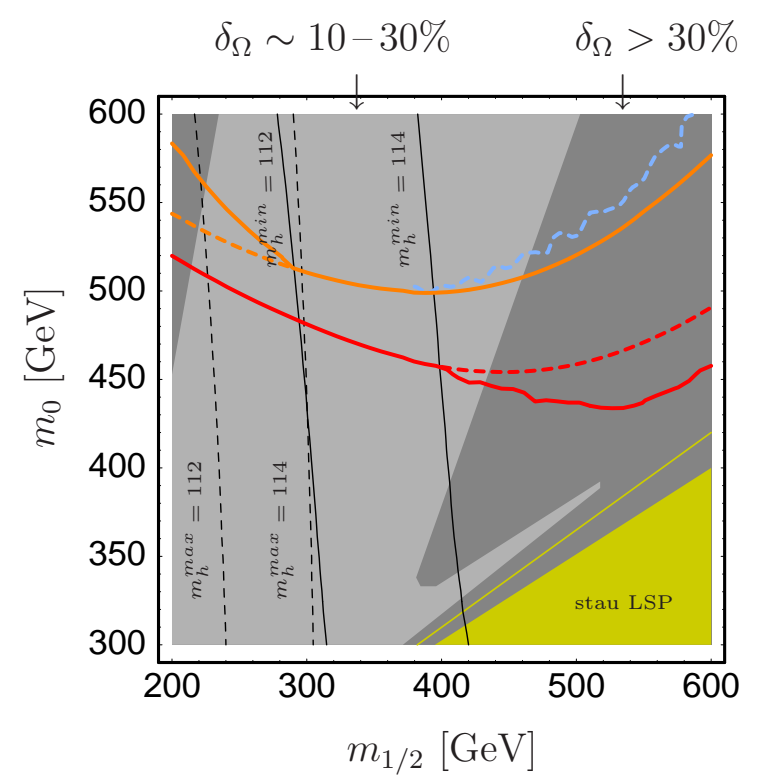

Figure 5: Comparison of results analogous to Fig. $2 \mathrm{~b}(\tan \beta=50)$ but for $A_{0}=m_{1 / 2}$. The red and orange lines show the variation of the bound $\Omega<0.1287$ due to differences in the spectra. The dashed red and orange lines show the situation when only comparing SOFTSUSY 1.9 and SPHENO 2.2.2. The gap between the dashed and the full red lines is due to a lighter $\tilde{\tau}_{1}$ and hence more $\tilde{\tau}$ co-annihilation in ISAJET 7.71; the gap between the dashed and the full orange lines is due to smaller $\tilde{\chi}_{1}^{0}$ and $A^{0}$ masses in ISAJET 7.71. The dashed blue line shows again how the maximal $\Omega$ moves when including SUSPECT 2.3.

\begin{tabular}{|c|c|c|c|c|}
\hline & ISAJET 7.71 & SOFTSUSY 1.9 & SPHENO 2.2.2 & SUSPECT 2.3 \\
\hline$\tilde{\chi}_{1}^{0}$ & 161.4 & 164.9 & 164.4 & 164.9 \\
\hline$\tilde{\tau}_{1}$ & 165.4 & 181.5 & 177.4 & 187.5 \\
\hline$\tilde{e}_{R}$ & 406.7 & 406.0 & 406.6 & 408.3 \\
\hline$h^{0}$ & 118.9 & 115.9 & 116.4 & 116.1 \\
\hline$A^{0}$ & 427.3 & 422.0 & 427.6 & 418.0 \\
\hline$m_{\tilde{\tau}_{1}}-m_{\tilde{\chi}_{1}^{0}}$ & 4.0 & 16.6 & 13.0 & 22.6 \\
\hline$m_{A}-2 m_{\tilde{\chi}_{1}^{0}}$ & 104.5 & 92.2 & 98.8 & 88.2 \\
\hline$\tilde{\chi}_{1}^{0} \tilde{\chi}_{1}^{0} \rightarrow b b$ & $3 \%$ & $45 \%$ & $30 \%$ & $66 \%$ \\
\hline$\tilde{\chi}_{1}^{0} \tilde{\chi}_{1}^{0} \rightarrow \tau \tau$ & - & $9 \%$ & $7 \%$ & $12 \%$ \\
\hline$\tilde{\chi}_{1}^{0} \tilde{\tau}_{1} \rightarrow h \tau$ & $21 \%$ & $17 \%$ & $24 \%$ & $7 \%$ \\
\hline$\tilde{\chi}_{1}^{0} \tilde{\tau}_{1} \rightarrow \gamma / Z \tau$ & $11 \%$ & $10 \%$ & $14 \%$ & $4 \%$ \\
\hline$\tilde{\tau}_{1} \tilde{\tau}_{1} \rightarrow b \bar{b}$ & $8 \%$ & $6 \%$ & $7 \%$ & $3 \%$ \\
\hline$\tilde{\tau}_{1} \tilde{\tau}_{1} \rightarrow h h$ & $28 \%$ & $3 \%$ & $7 \%$ & - \\
\hline$\tilde{\tau}_{1} \tilde{\tau}_{1} \rightarrow W W, Z Z, \gamma \gamma$ & $15 \%$ & $1 \%$ & - & - \\
\hline$\Omega$ & 0.017 & 0.107 & 0.081 & 0.136 \\
\hline
\end{tabular}

Table 7: Relevant masses and mass differences (in $\mathrm{GeV}$ ), the most important contributions and the resulting $\Omega$ for $m_{0}=376 \mathrm{GeV}, m_{1 / 2}=400 \mathrm{GeV}, A_{0}=-400 \mathrm{GeV}, \tan \beta=50$ and $\mu>0$. The higgsino fraction $f_{H}\left(\tilde{\chi}_{1}^{0}\right)$ is $0.8 \%$. 


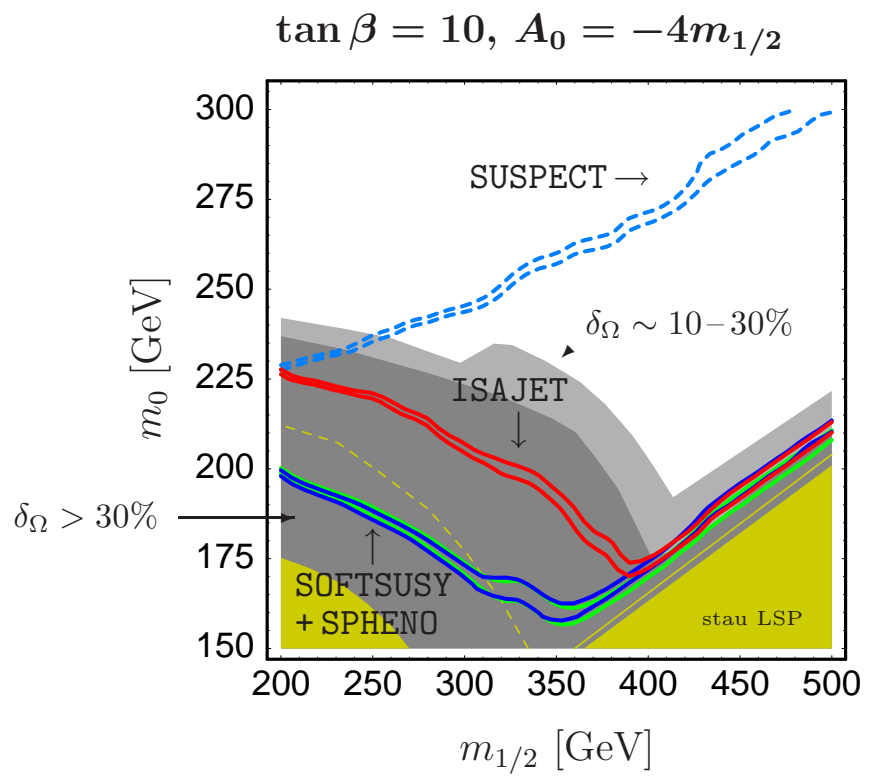

Figure 6: WMAP strips from the four public codes for $A_{0}=-4 m_{1 / 2}, \tan \beta=10, \mu>0$ and $m_{t}=175 \mathrm{GeV}$. The yellow region in the bottom right corner is excluded due to a $\tilde{\tau}_{1}$ LSP. In the yellow bottom left region, SOFTSUSY 1.9 and SPHENO 2.2.2 have a $\tilde{t}_{1}$ LSP; the yellow dashed line shows the bound of $\tilde{t}_{1}$ LSP in ISAJET 7.71.

\begin{tabular}{|c|c|c|c|c|}
\hline & ISAJET 7.71 & SOFTSUSY 1.9 & SPHENO 2.2.2 & SUSP \\
\hline$\overline{\tilde{\chi}_{1}^{0}}$ & 18140.8 & 14143.2 & 142.5 & 143.0 \\
\hline$\tilde{\tau}_{1}$ & 156.1 & 157.8 & 158.9 & 160.7 \\
\hline$\tilde{t}_{1}$ & 153.7 & 173.3 & 172.7 & 109.7 \\
\hline$h^{0}$ & 108.8 & 114.1 & 115.6 & 108.3 \\
\hline$m_{\tilde{\tau}_{1}}-m_{\tilde{\chi}_{1}^{0}}$ & 15.3 & 14.6 & 16.4 & 17.7 \\
\hline$m_{\tilde{t}_{1}}-m_{\tilde{\chi}_{1}^{0}}$ & 12.9 & 30.1 & 30.2 & -33.3 \\
\hline$\tilde{\chi}_{1}^{0} \tilde{\chi}_{1}^{0} \rightarrow e e, \mu \mu$ & - & $18 \%$ & $16 \%$ & - \\
\hline$\tilde{\chi}_{1}^{0} \tilde{\chi}_{1}^{0} \rightarrow \tau \tau$ & - & $22 \%$ & $19 \%$ & - \\
\hline$\tilde{\chi}_{1}^{0} \tilde{\chi}_{1}^{0} \rightarrow \gamma / Z \tau$ & - & $14 \%$ & $10 \%$ & - \\
\hline$\tilde{\chi}_{1}^{0} \tilde{t}_{1} \rightarrow$ th & $28 \%$ & $30 \%$ & $36 \%$ & - \\
\hline$\tilde{\chi}_{1}^{0} \tilde{t}_{1} \rightarrow \operatorname{tg}$ & $4 \%$ & $6 \%$ & $7 \%$ & - \\
\hline$\tilde{\chi}_{\underset{\sim}{\sim}}^{0} \tilde{t}_{1} \rightarrow Z t / W b$ & $2 \%$ & $4 \%$ & $4 \%$ & - \\
\hline$\tilde{t}_{1} \tilde{t}_{1} \rightarrow g g$ & $4 \%$ & - & - & - \\
\hline$\tilde{t}_{1} \tilde{t}_{1} \rightarrow g h$ & $2 \%$ & - & - & - \\
\hline$\tilde{t}_{1} \tilde{t}_{1} \rightarrow h h$ & $57 \%$ & $2 \%$ & $3 \%$ & - \\
\hline$\Omega$ & 0.004 & 0.116 & 0.120 & - \\
\hline
\end{tabular}

Table 8: Relevant masses and mass differences (in $\mathrm{GeV}$ ), the most important contributions and the resulting $\Omega$ for $m_{0}=161 \mathrm{GeV}, m_{1 / 2}=350 \mathrm{GeV}, A_{0}=-1400 \mathrm{GeV}, \tan \beta=10$ and $\mu>0 . f_{H}\left(\tilde{\chi}_{1}^{0}\right) \simeq 0.4 \%$. 
the very large mass splitting of the stops. Much larger discrepancies are however found when comparing with SUSPECT 2.3. Since SUSPECT 2.3 does not have the 2-loop RGEs for the squark parameters, including $A_{t}$, it predicts a much lighter $\tilde{t}_{1}$ than the other three programs. For the point of Table 8 , the difference in $m_{\tilde{t}_{1}}$ is about $60 \mathrm{GeV}$, or $35 \%$, making $\tilde{t}_{1}$ the LSP in the SUSPECT 2.3 spectrum. The large discrepancy between SUSPECT 2.3 and the other programs can be seen clearly in Fig. 6. Again, the results of SUSPECT 2.3 are reproduced by using only 1-loop RGEs for squark and slepton parameters in SOFTSUSY 1.9. Last but not least notice also that for the parameters of Table 8, in SOFTSUSY 1.9 and SPHENO 2.2.2 even though $\tilde{\tau}_{1}$ is the NLSP, coannihilation channels with $\tilde{t}_{1}$ dominate.

In summary, at very large $A_{0}<0$ one can get phenomenologically very different scenarii for the same mSUGRA point; it is clear that including the full two-loop RG running plus a careful treatement of threshold corrections is important for a reliable prediction of the relic density.

In this context it is also interesting to compare with the results of [11]. The 'best fit' points in their Fig. 15 are ${ }^{5} m_{0}=60 \mathrm{GeV}, m_{1 / 2}=300 \mathrm{GeV}, A_{0}=300 \mathrm{GeV}$ for $\tan \beta=10$ and $m_{0}=550 \mathrm{GeV}, m_{1 / 2}=500 \mathrm{GeV}, A_{0}=1280 \mathrm{GeV}$ for $\tan \beta=50$, both obtained with $m_{t}=178 \mathrm{GeV}$ and $m_{b}\left(m_{b}\right)=4.25 \mathrm{GeV}$ [32]. Both are scenarii of stau co-annihilation. The relevant masses, mass differences and the resulting values for $\Omega$ of Ref. [11] (SSARD) are given in Tables 9 and 10 together with the predictions from SOFTSUSY 1.9, SPHENO 2.2.2 and SUSPECT 2.3; we leave out ISAJET 7.71 where one cannot adjust $m_{b}\left(m_{b}\right)$. For the point with $\tan \beta=10$, the $\tilde{\chi}_{1}^{0}$ and $\tilde{\tau}_{1}$ masses of SSARD, which has full 2-loop RGEs, are roughly $2 \%$ higher than those of SOFTSUSY 1.9 and SPHENO 2.2.2. The $\tilde{\chi}_{1}^{0}-\tilde{\tau}_{1}$ mass difference and consequently also $\Omega$ lie within the values of SOFTSUSY 1.9 and SPHENO 2.2.2. For the point with $\tan \beta=50$, however, only SSARD has a viable spectrum with a neutralino LSP, while the three public codes get a $\tilde{\tau}_{1} \mathrm{LSP}$, about 40-60 GeV lighter than the $\tilde{\tau}_{1}$ in SSARD. Note also the $\sim 10 \%$ heavier $m_{A}$ from SSARD as compared to the public codes. We can recover a similar $\Delta M\left(\tilde{\chi}_{1}^{0} \tilde{\tau}_{1}\right)$ as [11] with SOFTSUSY 1.9 and SPHENO 2.2.2 for $A_{0}=1170 \mathrm{GeV}$. In this case we get $m_{\tilde{\chi}_{1}^{0}}=205$ $206 \mathrm{GeV}, m_{\tilde{\tau}_{1}}=218-222 \mathrm{GeV}, m_{A}=500-510 \mathrm{GeV}$ and $\Omega \simeq 0.098$. However, the fact remains that at large $\tan \beta$ (and large $A_{0}$ ) there are sizeable differences between SSARD and the public codes.

\section{Online comparison}

For an easy and user-friendly comparison of SUSY spectrum codes, we have set up a web application at

http://cern.ch/kraml/comparison/

Here the user can input mSUGRA parameter points in a web form. The value of the top-quark mass is also taken as an input while $m_{b}\left(m_{b}\right)$ and $\alpha_{s}$ are fixed to the values hardcoded in ISAJET. The mass spectra are then calculated by the latest versions of ISAJET, SOFTSUSY, SPHENO and SUSPECT and compared in an output table. The corresponding values for $\Omega, \delta \rho, \delta a_{\mu}, B(b \rightarrow s \gamma)$ and $B\left(b \rightarrow s \mu^{+} \mu^{-}\right)$are calculated with micrOMEGAs and also given in the table. SOFTSUSY is used with the option of full 2-loop running, as in this paper. For technical reasons, for the computation of $\Omega$ a 'static' version of micrOMEGAs

\footnotetext{
${ }^{5}$ Note that Ref. [11] uses the opposite sign convention for the trilinear $A$ couplings!
} 


\begin{tabular}{c||cccc} 
& SSARD & SOFTSUSY 1.9 & SPHENO 2.2.2 & SUSPECT 2.3 \\
\hline \hline$\tilde{\chi}_{1}^{0}$ & 119.4 & 117.7 & 117.4 & 117.8 \\
$\tilde{\tau}_{1}$ & 129.1 & 126.1 & 127.2 & 129.5 \\
$h^{0}$ & 113.9 & 111.7 & 112.0 & 111.8 \\
$m_{A}$ & 419.4 & 428.5 & 431.2 & 431.5 \\
\hline$m_{\tilde{\tau}_{1}}-m_{\tilde{\chi}_{1}^{0}}$ & 9.7 & 8.4 & 9.8 & 11.7 \\
$m_{A}-2 m_{\tilde{\chi}_{1}^{0}}$ & 181 & 193 & 196 & 196 \\
\hline$\Omega$ & 0.103 & 0.092 & 0.109 & 0.129 \\
\hline
\end{tabular}

Table 9: Relevant masses and mass differences (in $\mathrm{GeV}$ ) and the resulting $\Omega$ for $m_{0}=$ $60 \mathrm{GeV}, m_{1 / 2}=300 \mathrm{GeV}, A_{0}=300 \mathrm{GeV}, \tan \beta=10, \mu>0, m_{t}=178 \mathrm{GeV}$ and $m_{b}\left(m_{b}\right)=4.25 \mathrm{GeV}$.

\begin{tabular}{c||cccc} 
& SSARD & SOFTSUSY 1.9 & SPHENO 2.2.2 & SUSPECT 2.3 \\
\hline \hline$\tilde{\chi}_{1}^{0}$ & 211 & 206 & 205 & 206 \\
$\tilde{\tau}_{1}$ & 226 & 167 & 163 & 185 \\
$h^{0}$ & 117 & 116 & 116 & 116 \\
$m_{A}$ & 553 & 495 & 504 & 490 \\
\hline$m_{\tilde{\tau}_{1}}-m_{\tilde{\chi}_{1}^{0}}$ & 15 & -39 & -42 & -21 \\
\hline$\Omega$ & 0.119 & - & - & - \\
\hline
\end{tabular}

Table 10: Relevant masses and mass differences (in $\mathrm{GeV}$ ) for $m_{0}=550 \mathrm{GeV}, m_{1 / 2}=$ $500 \mathrm{GeV}, A_{0}=1280 \mathrm{GeV}, \tan \beta=50, \mu>0, m_{t}=178 \mathrm{GeV}$ and $m_{b}\left(m_{b}\right)=4.25 \mathrm{GeV}$.

is used which is limited to (co)annihilation channels initiated by $\tilde{\chi}_{1,2,3}^{0}, \tilde{\chi}_{1}^{ \pm}, \tilde{e}_{R}, \tilde{\mu}_{R}, \tilde{\tau}_{1}$, and $\tilde{t}_{1}$. We have checked that this is largely sufficient within mSUGRA. The webpage is also useful for comparisons with other spectrum codes and/or programs computing the neutralino relic density.

\section{Conclusions}

We have investigated the impact of uncertainties in SUSY spectrum computations on the prediction of the neutralino relic density. To this aim we have compared the results of four public spectrum codes, ISAJET 7.71, SOFTSUSY 1.9, SPHENO 2.2.2 and SUSPECT 2.3, in the context of mSUGRA. For 'moderate', i.e. not extreme, values of the model parameters, we found that the codes in general agree quite well, at the level of few percent, for the prediction of the SUSY spectrum. This is also true at large $\tan \beta$.

Nevertheless these small discrepancies can have a large impact on the prediction of the relic density of dark matter. We have studied in detail the most important scenarios for neutralino (co)annihilation. In the bulk region (although largely excluded by the LEP bound on $m_{h}$ ), predictions are under control, that is uncertainties are below the experimental uncertainties of WMAP. In the co-annihilation region, however, the uncertainties can easily exceed $30 \%$. Most of this is related to the mass difference between $\tilde{\tau}_{1}$ and the LSP. For this estimate of uncertainties we have used the predictions from ISAJET 7.71, 
SOFTSUSY 1.9 and SPHENO 2.2.2. SUSPECT 2.3, which only has 1-loop RG running for the sfermion mass parameters as opposed to full 2-loop RG running in the other codes, typically finds a higher $\tilde{\tau}_{1}$ mass. To reduce the uncertainty originating from the spectrum calculation to a level below the experimental uncertainty of WMAP, one needs a precision in the $\tilde{\chi}_{1}^{0}-\tilde{\tau}_{1}$ mass difference of the level of $1 \mathrm{GeV}$. This corresponds to computing the LSP and NLSP masses to per-mille accuracy. Already it has been shown that going from 2-loop to 3-loop RGE running [33] induces corrections of about that level.

Similar arguments hold for scenarios where the neutralinos annihilate through pseudoscalar exchange. Typically this means an enhanced coupling to the pseudoscalar, that is large $\tan \beta$. The critical parameter in this case is the $m_{A}-2 m_{\tilde{\chi}_{1}^{0}}$ mass difference. Although the codes we compared agree at the level of few percent on the pseudoscalar mass, this difference together with the difference in the LSP mass can again add up to $30 \%$ or more uncertainty in the relic density. To improve the precision of $m_{A}-2 m_{\tilde{\chi}_{1}^{0}}$, one not only needs to go to higher orders in the RG running but also a more precise treatment of the Yukawa couplings, especially of $h_{b}$, is needed eventually including the full 2-loop corrections. At this level also a precise treatement of $h_{\tau}$ becomes important. Notice, however, that the dominant source of uncertainty in $m_{A}$ is still the present error in the extraction of $m_{b}\left(m_{b}\right)$.

Models with non-zero $A_{0}$ have usually similar features w.r.t. the relic density as models with $A_{0}=0$. The exception is the case of a very large $A_{0}$ where the $\tilde{t}_{1}$ becomes light enough to contribute to co-annihilations. The existing $\sim 10 \%$ uncertainty in the prediction of the $\tilde{t}_{1}$ mass can then lead to order-of-magnitude discrepancies in the prediction of the relic density. In particular, ISAJET 7.71 predicts a lighter $\tilde{t}_{1}$ than SOFTSUSY 1.9 and SPHENO 2.2.2, and thus a much lower value for $\Omega$. The prediciton of SUSPECT 2.3 for $m_{\tilde{t}_{1}}$ is much below that of the other programs. In fact, in the $\tilde{t}_{1}$ co-annihilation region of SOFTSUSY 1.9 and SPHENO 2.2.2 (but also in the one of ISAJET 7.71), SUSPECT 2.3 does not provide a viable spectrum due to a $\tilde{t}_{1}$ LSP. This underlines the importance of including the full 2-loop RG running in the sfermion masses.

The picture is however different for extreme scenarios with very large $m_{0}$. These are the most difficult models to handle, and large discrepancies in the prediction of the spectrum calculators are found. This is especially the case for one of the most important parameters for the calculation of the relic density, $\mu$, which determines the masses and higgsino fractions of the neutralinos. Predictions for $\mu$ can vary by a factor of 2 or more, inducing huge order-of-magnitude differences in the relic density. An improvement of the situation requires in particular a much more precise computation of the top Yukawa coupling. A precise measurement of the top-quark mass, as addressed in [34], would also reduce the uncertainty. Owing to the extreme sensitivity of $\mu$ to the exact value of $h_{t}$ near the border of REWSB, we consider this region as very unstable.

We conclude that when using the WMAP bound for constraining mSUGRA models, uncertainties from the spectrum computation should be taken into account in addition to the experimental uncertainty of $\Omega$. For an estimate of the theoretical uncertainties one may use the maximal and minimal exclusion curves of different state-of-the-art codes, as we have done in this paper. The $\delta \Omega$ obtained this way is comparable to the one obtained in [18] by varying the renormalization scale within a given spectrum code. Finally, this theoretical uncertainty should also be combined with the uncertainty arising from the SM input parameters.

In parameter regions where $\delta \Omega$ originating from spectrum uncertainties is at present 
larger than the experimental uncertainty from WMAP, more precise calculations are certainly desirable to improve the reliability of relic density predictions within GUT-scale models. Such improvements will be even more important in view of the precision envisaged by the PLANCK experiment.

\section{Acknowledgements}

We thank Ben Allanach, Fawzi Boudjema and Werner Porod for useful discussions. We also thank Sven Heinemeyer and Keith Olive for providing the detailed results of SSARD. This work was supported in part by GDRI-ACPP of CNRS and by grants from the Russian Federal Agency for Science, NS-1685.2003.2. The work of S.K. is financed by an APART fellowship of the Austrian Academy of Sciences.

\section{Note added}

After this paper has appeared as a preprint on hep-ph, a new version of SUSPECT, v2.3.4, was published including the 2-loop RGEs for squark and slepton parameters. Owing to this improvement, the sfermion masses obtained with SUSPECT 2.3.4 agree well with those of SOFTSUSY 1.9, the two programs now being on the same level in the implementation of radiative corrections. In particular, for the $\tilde{t}$ co-annihilation point of Table 8, SUSPECT 2.3.4 now gives a viable spectrum similar to that of SOFTSUSY 1.9 or SPHENO 2.2.2, with $m_{\tilde{\chi}_{1}^{0}}=143 \mathrm{GeV}, m_{\tilde{t}_{1}}=178 \mathrm{GeV}$ and $\Omega=0.153$. This confirms our observation of the importance of these 2-loop terms. We note, however, that this does not change the $\delta \Omega$ shown in the figures, since SUSPECT 2.3 was not taken into account for the estimate of uncertainties.

\section{References}

[1] C. L. Bennett et al., Astrophys. J. Suppl. 148 (2003) 1 [arXiv:astro-ph/0302207].

[2] D. N. Spergel et al., Astrophys. J. Suppl. 148 (2003) 175 [arXiv:astro-ph/0302209].

[3] J. L. Feng, eConf C0307282 (2003) L11 [arXiv:hep-ph/0405215].

[4] J. R. Ellis, K. A. Olive, Y. Santoso and V. C. Spanos, Phys. Lett. B 565 (2003) 176 [arXiv:hep-ph/0303043].

[5] H. Baer and C. Balazs, JCAP 0305 (2003) 006 [arXiv:hep-ph/0303114]; H. Baer, C. Balazs and A. Belyaev, JHEP 0203 (2002) 042 [arXiv:hep-ph/0202076].

[6] U. Chattopadhyay, A. Corsetti and P. Nath, Phys. Rev. D 68 (2003) 035005 [arXiv:hep-ph/0303201].

[7] S. Profumo and C. E. Yaguna, arXiv:hep-ph/0407036.

[8] E. A. Baltz and P. Gondolo, arXiv:hep-ph/0407039. 
[9] G. Bélanger, F. Boudjema, A. Cottrant, A. Pukhov and A. Semenov, arXiv:hepph/0407218.

[10] A. B. Lahanas and D. V. Nanopoulos, Phys. Lett. B 568 (2003) 55 [arXiv:hep$\mathrm{ph} / 0303130]$.

[11] J. R. Ellis, S. Heinemeyer, K. A. Olive and G. Weiglein, arXiv:hep-ph/0411216.

[12] See e.g., B. C. Allanach et al., arXiv:hep-ph/0403133; T. Plehn, arXiv:hep$\mathrm{ph} / 0410063$.

[13] F. E. Paige, S. D. Protopescu, H. Baer and X. Tata, arXiv:hep-ph/0312045.

[14] B. C. Allanach, Comput. Phys. Commun. 143 (2002) 305 [arXiv:hep-ph/0104145].

[15] W. Porod, Comput. Phys. Commun. 153 (2003) 275 [arXiv:hep-ph/0301101].

[16] A. Djouadi, J. L. Kneur and G. Moultaka, arXiv:hep-ph/0211331.

[17] G. Bélanger, F. Boudjema, A. Pukhov and A. Semenov, arXiv:hep-ph/0405253; G. Bélanger, F. Boudjema, A. Pukhov and A. Semenov, Comput. Phys. Commun. 149 (2002) 103 [arXiv:hep-ph/0112278].

[18] B. C. Allanach, G. Bélanger, F. Boudjema, A. Pukhov, arXiv:hep-ph/0410091; B. C. Allanach, G. Bélanger, F. Boudjema and A. Pukhov, arXiv:hep-ph/0410049.

[19] B. C. Allanach, S. Kraml and W. Porod, JHEP 0303 (2003) 016 [arXiv:hep$\mathrm{ph} / 0302102]$ and references therein.

[20] B. C. Allanach, G. Bélanger, F. Boudjema, A. Pukhov and W. Porod, arXiv:hep$\mathrm{ph} / 0402161$.

[21] P. Skands et al., JHEP 0407 (2004) 036 [arXiv:hep-ph/0311123].

[22] G. F. Giudice, E. W. Kolb and A. Riotto, Phys. Rev. D 64 (2001) 023508 [arXiv:hepph/0005123]; S. Khalil, C. Munoz and E. Torrente-Lujan, New J. Phys. 4, 27 (2002) [arXiv:hep-ph/0202139]; C. Pallis, Astropart. Phys. 21 (2004) 689 [arXiv:hep$\mathrm{ph} / 0402033]$.

[23] M. Joyce, Phys. Rev. D 55 (1997) 1875 [arXiv:hep-ph/9606223]; P. Salati, Phys. Lett. B 571 (2003) 121 [arXiv:astro-ph/0207396].

[24] D. M. Pierce, J. A. Bagger, K. T. Matchev and R. j. Zhang, Nucl. Phys. B 491 (1997) 3 [arXiv:hep-ph/9606211].

[25] B. C. Allanach, A. Djouadi, J. L. Kneur, W. Porod and P. Slavich, JHEP 0409 (2004) 044 [arXiv:hep-ph/0406166].

[26] L. V. Avdeev and M. Y. Kalmykov, Nucl. Phys. B 502 (1997) 419 [arXiv:hepph/9701308]. A. Bednyakov, A. Onishchenko, V. Velizhanin and O. Veretin, Eur. Phys. J. C 29 (2003) 87 [arXiv:hep-ph/0210258]. 
[27] M. Carena, D. Garcia, U. Nierste and C. E. M. Wagner, Nucl. Phys. B 577 (2000) 88 [arXiv:hep-ph/9912516].

[28] Joint LEP2 SUSY Working Group, http://lepsusy.web.cern.ch/lepsusy/

[29] A. Balbi, C. Baccigalupi, F. Perrotta, S. Matarrese, and N. Vittorio, Astrophys. J. 588 (2003) L5 [arXiv:astro-ph/0301192].

[30] SUSY Parameter Analysis project (SPA), http://spa.desy.de/spa/

[31] M. E. Gomez, T. Ibrahim, P. Nath and S. Skadhauge, Phys. Rev. D 70 (2004) 035014 [arXiv:hep-ph/0404025].

[32] S. Heinemeyer, K. Olive, private communication.

[33] I. Jack, D. R. T. Jones and A. F. Kord, Phys. Lett. B 579 (2004) 180 [arXiv:hep$\mathrm{ph} / 0308231]$.

[34] S. Heinemeyer, S. Kraml, W. Porod and G. Weiglein, JHEP 0309 (2003) 075 [arXiv:hep-ph/0306181]. 